Running head: RISK FACTORS FOR ASD

\title{
An Analysis of an Online Survey of Risk Factors and Severe Characteristics in Autism Spectrum Disorders
}

\begin{abstract}
A thesis submitted to the Faculty of Graduate Studies and Research in Partial Fulfillment of the requirements for the Master of Arts degree in Psychology
\end{abstract}

by

Robin Hicks

Department of Psychology

Carleton University

May, 2008

C2008 Robin Hicks 


$\begin{array}{ll}\begin{array}{l}\text { Library and } \\ \text { Archives Canada }\end{array} & \begin{array}{l}\text { Bibliothèque et } \\ \text { Archives Canada }\end{array} \\ \begin{array}{l}\text { Published Heritage } \\ \text { Branch }\end{array} & \begin{array}{l}\text { Direction du } \\ \text { Patrimoine de l'édition }\end{array} \\ \begin{array}{l}\text { 395 Wellington Street } \\ \text { Ottawa ON K1A ON4 } \\ \text { Canada }\end{array} & \begin{array}{l}\text { 395, rue Wellington } \\ \text { Ottawa ON K1A 0N4 } \\ \text { Canada }\end{array}\end{array}$

Your file Votre référence ISBN: 978-0-494-43462-8

Our file Notre référence

ISBN: 978-0-494-43462-8

NOTICE:

The author has granted a nonexclusive license allowing Library and Archives Canada to reproduce, publish, archive, preserve, conserve, communicate to the public by telecommunication or on the Internet, loan, distribute and sell theses worldwide, for commercial or noncommercial purposes, in microform, paper, electronic and/or any other formats.

The author retains copyright ownership and moral rights in this thesis. Neither the thesis nor substantial extracts from it may be printed or otherwise reproduced without the author's permission.
AVIS:

L'auteur a accordé une licence non exclusive permettant à la Bibliothèque et Archives Canada de reproduire, publier, archiver, sauvegarder, conserver, transmettre au public par télécommunication ou par l'Internet, prêter, distribuer et vendre des thèses partout dans le monde, à des fins commerciales ou autres, sur support microforme, papier, électronique et/ou autres formats.

L'auteur conserve la propriété du droit d'auteur et des droits moraux qui protège cette thèse. $\mathrm{Ni}$ la thèse ni des extraits substantiels de celle-ci ne doivent être imprimés ou autrement reproduits sans son autorisation.
In compliance with the Canadian

Privacy Act some supporting forms may have been removed from this thesis.

While these forms may be included in the document page count, their removal does not represent any loss of content from the thesis.
Conformément à la loi canadienne sur la protection de la vie privée, quelques formulaires secondaires ont été enlevés de cette thèse.

Bien que ces formulaires aient inclus dans la pagination, il n'y aura aucun contenu manquant.

\section{Canada}




\begin{abstract}
The present study analyzed the responses of 918 parents of children with an Autism Spectrum Disorder (ASD) to an online survey. Risk factors, severe characteristics and differences among groups were examined. Comorbid medical conditions, family history of ASD and mental illness, obstetric complications and parental age over 35 were commonly reported. Comorbid medical conditions, family history of ASD and mental illness, birth order, gestational age at birth and total number of risks were related to specific diagnostic categories. Self-injury, self-stimulation, and sleep disorders were frequently reported. Several risk factors were significantly related to severe characteristics. Gender was related to severity of self-injury and sleep disorders. Risks did not differ based on where families live in this mostly North American sample. Results support multiple etiologies primarily based on genetic risks. Implications, limitations, and future directions for research are discussed.
\end{abstract}




\section{Acknowledgements}

I am grateful to many people who have supported and inspired me throughout this journey of learning. First, I would like to thank my advisor and committee members for their guidance on this project. I am grateful to Anne Bowker, $\mathrm{PhD}$., for providing the opportunity to work with this valuable data set and for providing academic guidance and moral support. Your guidance and teaching style have inspired me in my own teaching career. To the members of my committee -- Jo-Anne LeFevre, PhD., Jennifer Dunn Geier, $\mathrm{PhD}$., Robert Leblanc, $\mathrm{PhD}$., and Bernadette Campbell, $\mathrm{PhD}$., I appreciated your input and expert guidance. I would like to express special thanks to Dr. Jennifer Dunn Geier, for continuous encouragement over the years to pursue my personal goals. I consider myself fortunate to have had the opportunity to learn from each of you.

My family and friends have been a source of constant support and encouragement throughout my studies. Thank you to my husband, Stephen, for your support and patience. To my children, Nathan and Hunter -- You are the light of my life and I hope you will forgive your mother for the hours spent at the computer! I owe special thanks to my parents, siblings, extended family, friends and colleagues who have been a constant source of encouragement and believed in my success every step of the way.

Finally, I wish to express my appreciation to the families and individuals affected by autism, whose courage to face everyday, despite many challenges, inspires me to continue learning and contributing to the field of autism with determination and hope for a brighter future. 
Table of Contents

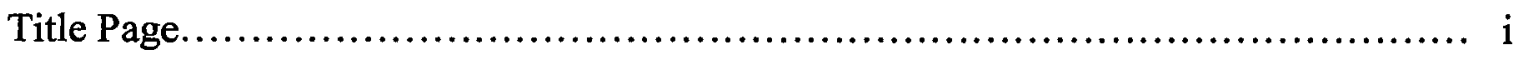

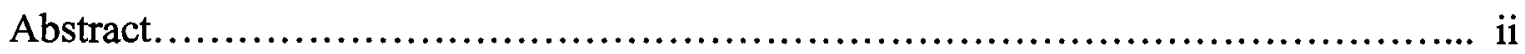

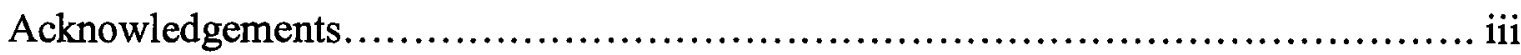

Table of Contents......................................................................... iv

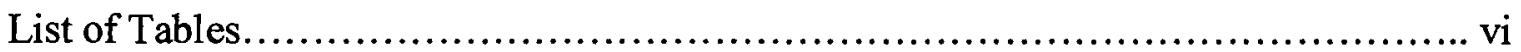

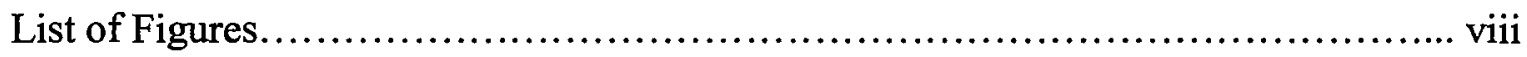

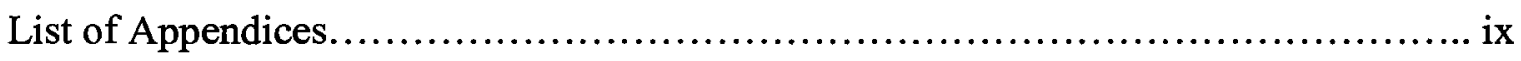

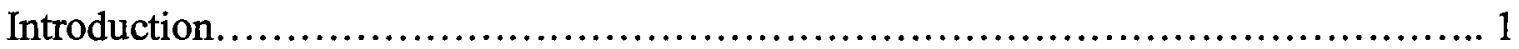

Autism Spectrum Disorders..............................................

Psychological Models of Autism..........................................6

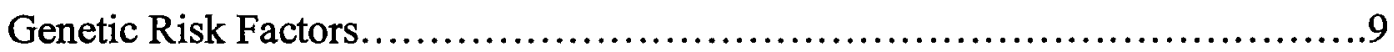

Parental Age............................................................

Social Class......................................................................

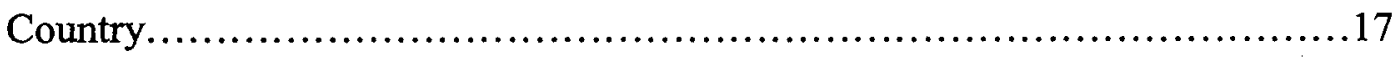

Gender................................................................... 17

Birth Order.................................................................

Environmental Risk Factors..............................................19

Obstetric Complications....................................................19

Severe Characteristics.....................................................22

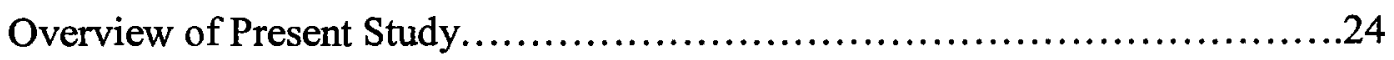

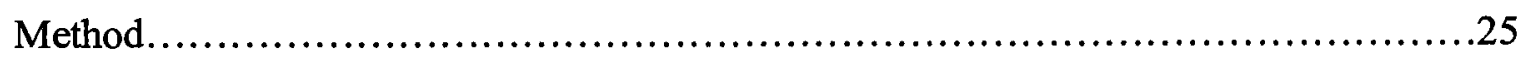

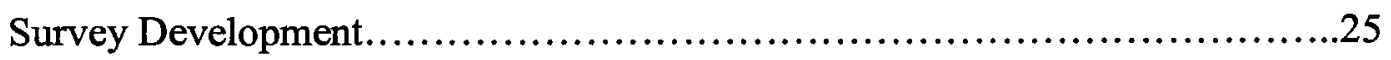




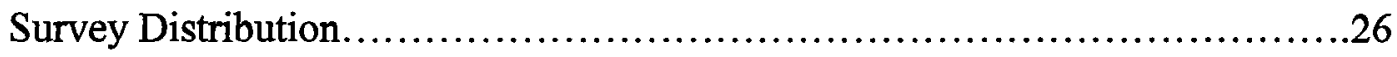

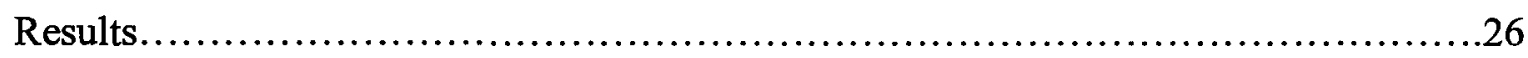

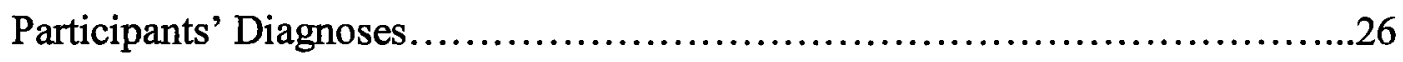

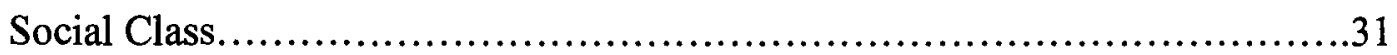

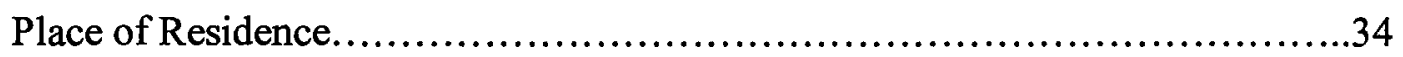

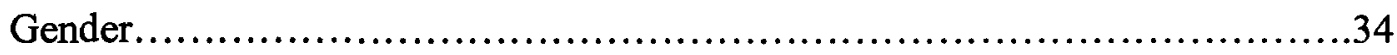

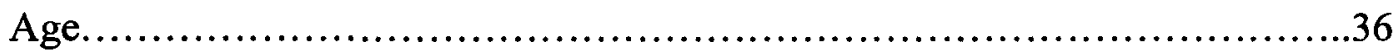

Associated Medical Conditions.............................................40

Other Cases of ASD in Family ..............................................44

Family History of Mental Illness.............................................47

Parental Age................................................................... 49

Birth Order and Family Size................................................51

Obstetric Complications....................................................54

Number of Risk Factors related to each Disorder..............................57

Severe Characteristics...................................................60

Self-injurious Behaviours...........................................6 60

Self-stimulatory Behaviours.......................................62

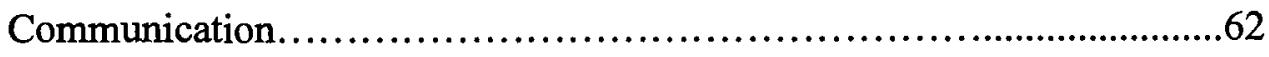

Sleep Problems.....................................................64

Risk Factors related to Severe Characteristics.........................67

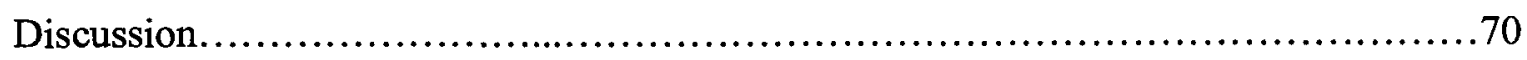

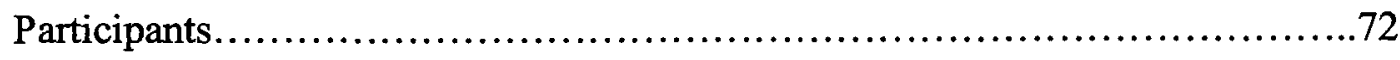

Risk Factors.................................................................... 
Risk Factors in ASD

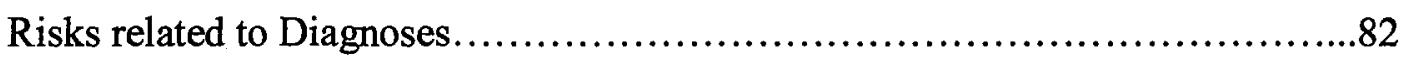

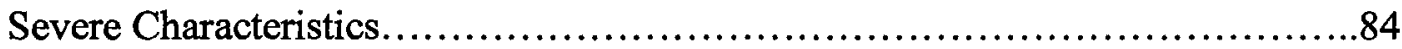

Risk Factors related to Severe Characteristics................................87

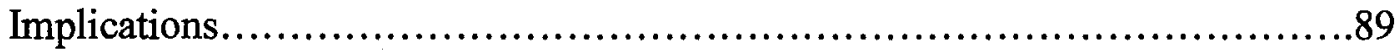

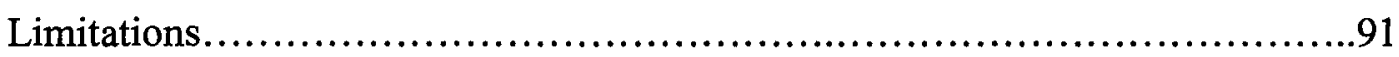

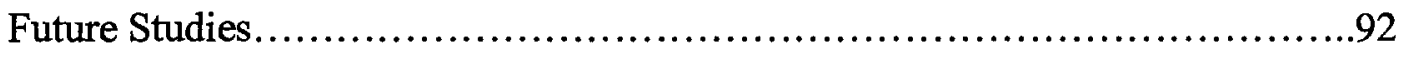

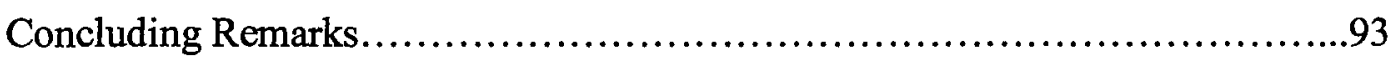

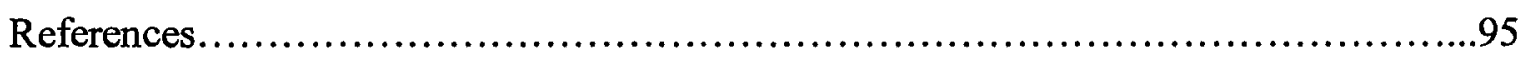

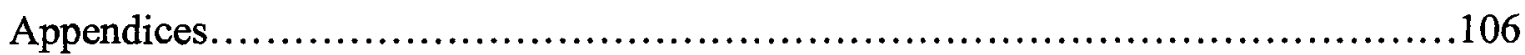


List of Tables

Table 1. Participants' Diagnoses.................................................29

Table 2. Nature of Change of Diagnosis........................................ 30

Table 3. Family Average Annual Income..........................................32

Table 4. Occupation of Parents..............................................33

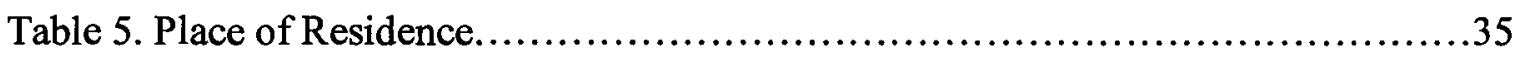

Table 6. Age of Child with ASD at time of Survey Completion........................37

Table 7. Age of Child with ASD at time of Diagnosis................................38

Table 8. Type of Medical Condition Reported Across All Groups.....................42

Table 9. Comparison of Comorbid Medical Condition Types by Diagnosis..............43

Table 10. Number of Other Cases of ASD in Family .............................46

Table 11. Number of Relatives with History of Mental Illness........................48

Table 12. Age of Parents at Time of Child's Birth................................50

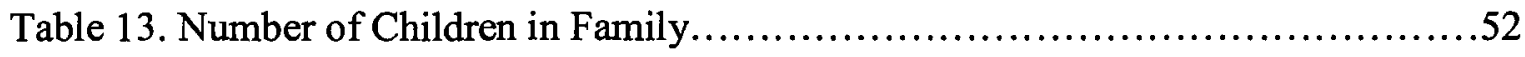

Table 14. Number of Risk Factors per Group....................................58

Table 15. Primary Type of Communication System by Diagnosis....................63

Table 16. Presence of Sleep Disorders in each Diagnostic Group......................66

Table 17. Summary of Relationships Between Risk Factors and Severe Characteristics...68 


\section{List of Figures}

Figure 1. Percent of Group Diagnosed at Specified Age................................39

Figure 2. Birth Order within Diagnostic Groups....................................53

Figure 3. Categories of Obstetric Complications for all Individuals with ASD...........55

Figure 4. Gestational Age at Birth in Weeks.....................................56

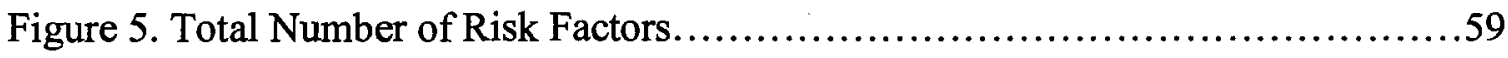

Figure 6. Severity of Self-Injurious Behaviour Within Groups......................61

Figure 7. Percentage of ASD Cases with Specific Types of Sleep Disorders..............65 


\section{List of Appendices}

Appendix A. Internet Survey......................................................................................106

Appendix B. Hours of Assessment by Diagnostic Group..............................115

Appendix C. Crosstabulation Results of Place of Residence by Risk Factors.............117

Appendix D. Crosstabulation Results of Gender by Risk Factors.......................118

Appendix E. Crosstabulation Results of Diagnosis by Birth Order.....................119 
An Analysis of an Online Survey of Risk Factors and Severe Characteristics in Autism Spectrum Disorders

Despite nearly 75 years of history as a recognized disorder, the cause of autism is still a mystery. Researchers have identified the source of dysfunctional characteristics of autism and it is now known to be a disorder of neurobiological origin. However, there is an ongoing debate as to the specific causal pathways leading to the expression of the disorder. Many researchers argue that autism has not one, but multiple possible etiologies. In the current study, I reviewed the three specific diagnostic categories that comprise the broader classification of Autism Spectrum Disorders (ASD), introduced three major psychological models of ASD, discussed the evidence related to the possible inheritance of the disorder and discussed the likelihood that other non-genetic factors may contribute to its development. Archival data from an online survey of parents of children with an ASD were analyzed.

The purpose of the present study was twofold. First, I examined the frequency that parents reported their children with ASD experienced various genetic and other risk factors and investigated relationships between these factors, and the three diagnostic categories of ASD. The identification of risk factors may help to ensure that children with ASD are identified and begin effective intervention as early as possible. Early intervention is currently viewed as being critical to a positive outcome. Second, I reported the frequency of parent-reported severe characteristics and extended the current body of literature on risk factors by investigating possible relationships between risk factors and specific, severe characteristics within this disorder. Here again, the early identification of children at risk for severe behavioural manifestations of the disorder could lead to early 
access to and prioritization for services. A particular emphasis was placed on identifying the prevalence of various risk factors and severe characteristics within this sample as these have not been extensively reported in the literature, and particularly not in such a large and diverse sample.

Autism Spectrum Disorders

In 1943, Leo Kanner used the term "infantile autism" to describe eleven children primarily suffering from social deficits, impaired communication and accompanying behavioural oddities but for whom intellect and motor development were generally intact. Over the years, the use of the term and understanding of the disorder has evolved but the core characteristics of the disorder have remained consistent with Kanner's initial observations. Autism is characterized by severe and prolonged impairment in social interaction, communication deficits and patterns of behaviour and interests that are restricted and/or repetitive (DSM-IV-TR; American Psychiatric Association, 2000). Autism is classified as one of the Pervasive Developmental Disorders in the Diagnostic and Statistical Manual fourth edition - text revision (DSM-IV-TR; American Psychiatric Association, 2000). Five categories of disorders are included in the classification, including Asperger's Disorder, Autistic Disorder (or classic autism), Pervasive Developmental Disorder - Not Otherwise Specified (PDD-NOS or atypical autism), Rett's Disorder, and childhood disintegrative disorder. Rett's Disorder and Childhood Disintegrative Disorder differ in a variety of ways from the other three categories; most notably by the progressive deterioration of motor skills (Rutter, 2005; Volkmar \& Rutter, 1995) and are therefore not included in the present study. The remaining categories of Autistic disorder, Asperger's disorder and PDD-NOS are of particular interest in this study 
and as a group, will be referred to as autism spectrum disorders (ASD) throughout this paper.

In the 1960's autism had prevalence rates of 5/10 000 but due, at least in part, to increased parental and professional awareness of developmental problems, increased availability of intervention programs and changed diagnostic criteria, that rate is on the rise (Fombonne, 2005; Tidmarsh \& Volkmar, 2003). Once considered a rare disorder, recent studies have shown the prevalence of the combined ASDs is at least 36.4/10 000 using conservative estimates but $60 / 10000$ is likely more accurate (Fombonne, 2005). The male-to-female ratio in children is $4.3: 1$ (Fombonne, 2003) and this disorder exists across all educational and socioeconomic levels (Fombonne, 2003).

The three categories of ASDs share common characteristics and yet are arguably distinct. ASD is considered a lifelong disorder for $90 \%$ of individuals (Smith, 1999) and affects all aspects of an individual's functioning (Tidmarsh \& Volkmar, 2003). A diagnostic assessment should include evaluation by a multidisciplinary team to determine medical history, adaptive functioning, intelligence, communication skills and behavioural characteristics (Tidmarsh \& Volkmar, 2003). The diagnostic assessment typically involves the use of one or more autism specific observation tools such as the Childhood Autism Rating Scale (CARS, Schopler, Reichler \& Rochen-Renner, 1988), the Autism Diagnostic Interview-Revised (ADI-R; Lord, Rutter \& LeCouteur, 1994) or the Autism Diagnostic Observation Schedule-Generic (ADOS-G; Lord et al., 2000). Once diagnosed, some children who have received early intensive intervention based on the principles of applied behavioural analysis have made considerable progress and in some cases were no longer autistic (Lovaas, 1987; Smith, 1999). However, the longterm prognosis for 
individuals with ASD is poor in that nearly $80 \%$ of adults will remain significantly dependent on family and support services (Howlin et al., 2004). There is some evidence to suggest that individuals with Asperger's may have a slightly more positive outcome (Klin, McPartland \& Volkmar, 2005). Among clinicians and researchers, there is general consensus that a child who is diagnosed and begins treatment as early as possible in their development has a better chance of avoiding the negative consequences of the disorder (Guralnick, 1998; reviewed by Rogers, 1998). Most children with ASD are currently not identified until around three years of age when they demonstrate increasing deficits in social and communication development compared to their age peers (Filipek et al., 1999). Identification based on developmental assessment has shown that many children can be identified as early as 24 months (Landa \& Garrett-Mayer, 2006) and some suggest that developmental differences are detectable as early as the first year (Osterling et al., 2002). The identification of early signs of risk in the neonatal period including genetic or other familial factors and pre- and perinatal issues, may contribute significantly to the earlier identification of these children.

Autistic Disorder (AD) or "classical autism" is considered to be at the more severe end of the spectrum and recent studies estimate the prevalence to be between 13 and 16 $/ 10000$ (Fombonne, 2005). Half of all preschool children with autistic disorder are nonverbal (Smith, 1999) and cognitive functioning in individuals with autism is often impaired with as many as $70-80 \%$ of individuals meeting diagnostic criteria for mental retardation (Fombonne, 2003; Smith, 1999; Wodrich, 1997). To receive a diagnosis of $\mathrm{AD}$, delays in social development, language or symbolic play must be present before three years of age. A child with $\mathrm{AD}$ may present as being disinterested in others and appear as 
if they are in a world of their own. He/she may avoid eye contact and appear as though they are deaf because they are unresponsive to their name and do not follow instructions. The child may ritualistically stack or line up toys rather than playing with them as they are intended and may rock their body, flap their hands or appear overly sensitive to common sounds like vacuums or hairdryers. Children with this severe disorder are also prone to a variety of behavioural excesses including tantrums, aggression or self-injury.

Children who exhibit many of the characteristics of autism but don't meet the diagnostic criteria for other pervasive developmental disorders receive a diagnosis of PDD-NOS. Therefore, children who do not fit the diagnosis because of age of onset or don't present with some key features described in other categories or who have less severe symptoms would fit this diagnosis (Tidmarsh \& Volkmar, 2003). The clinical criteria for PDD-NOS have been criticized for being ambiguous, and thus leading to too many false positive diagnoses (2003). Based on a review of 37 studies, prevalence of PDD-NOS was estimated at 20.8/ 10000 and is therefore, more common than $\mathrm{AD}$ (Fombonne, 2005). Children in this diagnostic category are sometimes described as being 'high functioning' in that they have better communication compared to those with $\mathrm{AD}$ and/or have normal cognition (Fombonne, 2005).

Asperger's Disorder or Asperger's Syndrome (AS) was first described by an Austrian pediatrician, Hans Asperger, in 1944 (Frith, 1991; English translation). He described a small group of children who despite seemingly adequate cognitive and verbal skills were experiencing significant challenges with social integration. Today the diagnosis closely mirrors these observations. Similar to AD and PDD-NOS, this disorder is characterized by impaired social interaction and a restricted range of interests and 
activities. However, it is distinguished from these categories by a relative preservation of cognitive and communication skills in the first three years of life (Klin, McPartland \& Volkmar, 2005) and therefore, diagnosis tends to occur at a later age than for AD. As individuals tend to be 'high functioning', AS is considered to be at the least severe end of the autism spectrum. However, the lack of social competence can be quite debilitating in this group. These individuals are sometimes described as speaking like little professors with overly formal and sophisticated words. They may perseverate on a narrow range of conversation topics, preferring to talk about nothing else and this leads to social isolation. Although limited data is available, the rate of AS is estimated to be approximately onefifth that of autism for a conservative estimate of 2.6/10 000 (Fombonne, 2005). Psychological Models of Autism

Since the disorder was first identified, the cause of autism has been a source of speculation and controversial theories. In the late 1960's, Bruno Bettleheim theorized that autism was caused by cold and distant parents who were damaging the social capacity of their children (1967). This psychosocial theory was debunked decades ago yet many families were scarred by the shame and scorn of feeling responsible for their children's disability and perhaps most importantly, many children failed to receive any meaningful treatment. It is now commonly accepted that autism is a disorder of neurological origin. A variety of psychological models have been developed to explain the characteristics of the disorder. The recognition of high rates of seizure disorders in individuals with ASD pointed to brain involvement and as a result, Theory of Mind (Baron-Cohen, Leslie \& Frith, 1985), Weak Central Coherence (Frith, 1989) and Executive Functions (Duncan, 1986) are some of the most commonly investigated neurocognitive theories in autism 
(Volkmar \& Pauls, 2003). Each of these theories is briefly reviewed below in terms of the broader phenotype of autism and the possible inheritance of the disorder. The broader phenotype of autism has been used to describe a group of characteristics that are similar but milder than those that are central to the diagnosis of an ASD. These characteristics have been reported in as many as 1 in 5 first-degree relatives of individuals with autism (Rutter, 2005a).

Theory of Mind (Baron-Cohen, Leslie \& Frith, 1985) as it is applied to autism is also known as mindblindness (Baron-Cohen, 1995) and is based on the hypothesis that individuals with ASD lack the ability to understand the perspective of others. This 'mindblindness' results in the characteristic social difficulties associated with intersubjectivity and empathy that are common to all three classifications of the disorder, regardless of level of intellectual ability (Baron-Cohen et al., 2005). Parents of children with ASD have also shown mild deficits perspective-taking, indicating a possible broader cognitive phenotype of autism and therefore suggestive of a genetic susceptibility to the disorder (Baron-Cohen, 2001).

Executive Function (Duncan, 1986) is a construct used to describe a constellation of cognitive skills involving flexibility, planning, inhibition, organization, self-monitoring, goal-setting and working memory (Ozonoff, South \& Provencal, 2005). Deficits in these executive functions are purported to account for many of the attentional and behavioural characteristics of autism including adherence to strict routines, a need for sameness, difficulty in shifting attention and repetitive behaviours such as stereotypies. Executive dysfunction has been reported in individuals with ASD and also in their family members. These findings are suggestive of a broader autism phenotype and support the notion that 
cognitive characteristics, key to the disorder, may be inherited (Ozonoff, South \& Provencal, 2005).

Weak central coherence (Frith, 1989) is another cognitive theory of autism which attempts to account for some of the observed assets and 'splinter skills' or savant abilities in cognitive processing sometimes seen in autism. Central Coherence is a construct used to describe the tendency for an individual to pull together pieces of information to gain contextual meaning. In individuals with ASD, weak central coherence has been described as the tendency to focus on detail while missing the more global meaning or contextually relevant information (Frith, 1989). This attention to detail, or local processing, may account for some of the observed superior skills of some autistic individuals in puzzle completion, detailed drawing and so on, but also leads to their nonfunctional preoccupation with detail and parts of the whole and a the lack of global processing that leads to their inability to determine the gist or 'bigger picture' and also, to significant difficulties in recall of information (Frith, 1989). Happé (2005) proposes that this weak central coherence may be considered a cognitive style rather than a deficit. Weak central coherence has been observed in the fathers of children with autism which supports the notion of a broader autism phenotype and the possible inheritance of genetic traits related to the disorder (Briskman, Happé, \& Frith, 2001).

These cognitive theories each attempt to account for the characteristics expressed by individuals with ASD. ASD is a neurological disorder that is behaviourally manifested and thus we cannot ignore the developmental impact of the neurological deficits. For example, the characteristic tendency of children with autism to lack initiation of joint attention is likely to alter opportunities for social interaction and learning that may further 
impact development (Mundy \& Burnette, 2005). The disorder is commonly accepted to have multiple and/or complex etiologies and it is most likely the location of the neuropathology, rather than the pathology itself that is responsible for clinical presentation (Tharp, 2003). Furthermore, the reported neurocognitive differences in families of individuals with ASD, lends credibility to the notion of a broader phenotype of autism and therefore, the genetic inheritance of the disorder. It is important to note that those families which show evidence of the broader phenotype also have children who are not demonstrably affected by ASD. So, although genetic liability for the disorder is clearly a risk, other non-genetic factors that may trigger the genetic predisposition into a full-blown expression of the disorder must be considered.

Much has been learned about the manifestation of ASD characteristics and improvements have been made in assessment, diagnosis and treatment. Although many theories exist about the etiology of the disorder, the cause is still unknown. Empirical evidence supports some of these theories whereas others have mixed support or even consistently negative findings. However, the empirical evidence does support that this is a multifactor disorder in which the role of genetics plays an important part. In the present thesis, I reviewed the evidence related to both genetic and non-genetic risk factors for ASDs. The hypothesis on which this study was designed is that ASD may arise as the result of some combination of genetic predisposition and environmental influences and that some of the factors may be of greater risk for triggering the disorder than others. Genetic Risk Factors

The evidence to support the genetic basis for ASD comes from a variety of sources. A small number of genetic and medical conditions have been positively 
associated with ASD although a definitive causal role in the development of ASD is still unclear (Rutter, 2005a). An estimated 10\% of children with ASD have an identified neurodevelopmental genetic syndrome such as Fragile-X, Tuberous Sclerosis or neurofibromatosis (Rutter, 2005a; 2005b; Tharp, 2003). In a recent study of 100 boys with Asperger Syndrome, $8 \%$ had an identifiable medical syndrome or chromosomal abnormality (Gillberg \& Cederlund, 2005). Although studies have begun to identify particular chromosomes with strong associations to ASD, the significant difficulty in isolating a single gene responsible for the disorder suggests that multiple genes, a complex pattern of inheritance or possibly environmental insults may be responsible for the expression of the disorder (Rutter, 2005a; Volkmar, 2003). Chromosomes 2 and 7 and a few others have been targeted in recent studies as researchers attempted to identify the specific susceptibility genes for ASD (Rutter, 2005a; Volkmar \& Pauls, 2003). Possibly as many as $3-12$ susceptibility genes acting synergistically lead to the disorder and it is anticipated that researchers may be able to isolate these genes within the next decade (Rutter, 2005a).

Primary evidence of the role that genetics plays in ASD comes from twin and family studies. The heritability for ASD is estimated at an astounding 90\% (Rutter, 2005a, 2005b; Volkmar \& Pauls, 2003). In cases where medical/chromosomal cause had been ruled out, the concordance rate for ASD in monozygotic (identical) twins is approximately $60 \%$ as compared to $5 \%$ in dizygotic (fraternal) twins (Rutter, 2005a). Furthermore, when one child has ASD, their identical twin may not necessarily meet diagnostic criteria for ASD, but is more likely to express signs of the broader phenotype than is a fraternal twin (Rutter, 2005a, 2005b). Specifically, monozygotic twins have been found to be $90 \%$ 
concordant for the broader phenotype compared to only 1 in 10 dizygotic pairs (Rutter, 2005b).

The rate of ASD in families has also been investigated and the evidence supports genetic risk as well as the possibility that environmental risks may play a role. In a study of first-degree relatives, the rate of autism in siblings of individuals with autism was $3 \%$ with an additional $3 \%$ with a loosely defined ASD (Bolton et al, 1994). Family studies have consistently shown that the risk of recurrence of ASD is $2-7 \%$ which may seem low, however; the relative risk is $50-200$ times higher than would be expected in the general population (Volkmar \& Pauls, 2003). Furthermore, Rutter estimates that the broader phenotype occurs in $10-20 \%$ of first degree relatives of individuals with ASD (2005b). In a comparison of biological and nonbiological relatives, autistic characteristics were more common in biological family members lending further support to the likelihood of inheritance for the broader phenotype (Szatmari et al., 2000). Rutter speculates that some siblings develop the disorder while others do not because they have inherited only some of the relevant genes (2005a, 2005b). Alternatively, the existence of the broader phenotype may mean that an individual who has the susceptibility genes for ASD requires an additional risk factor(s) to trigger the full blown expression of the disorder (Rutter, 2005b). Similar support was found in a study of risk factors in individuals with Asperger Syndrome. Genetics and family history were indicated in $55 \%$ of the sample of 100 individuals with AS; some combination of genetics and pre or perinatal risk was evident in $11 \%$ and pre or perinatal risk alone was implicated in $13 \%$ of the sample (Gillberg \& Cederlund, 2005). The current study had a large, geographically diverse sample which provided the opportunity to investigate how common it was for an individual with ASD to 
have other relatives with ASD and to explore what some of the other possible risk factors may be. The risk factors associated with each diagnosis were also investigated.

Researchers have also been interested in trying to identify other disorders that run in families that may be related to ASD. In a case-control study of 115 individuals with autism in Denmark, psychiatric disorders were found in $15.7 \%$ of mothers of autistic children compared to $8.2 \%$ in the control group (Mouridsen, Rich, Isager \& Nedergaard, 2007). Personality disorder was the only diagnostic category significantly overrepresented in the sample with $7.8 \%$ of mothers affected compared to $2.1 \%$ of control group mothers (Mouridsen et al., 2007). In another Danish study, risk of autism was found to be twice as high in cases where there was a history of maternal psychiatric admission (Lauritsen, Pedersen \& Mortensen, 2005). In a study that included 99 individuals with ASD, affective disorders, motor tics and obsessive compulsive disorder were found significantly more often in relatives of autistic subjects as compared to relatives of children with Down's Syndrome (Bolton, Pickles, Murphy \& Rutter, 1998). Anxiety has also been found to be more common amongst relatives of individuals with ASD (Murphy et al., 2000). In a study of 79 participants with ASD, depression was found in $58 \%$ of the mothers in the sample and anxiety in 34\% (Mikali, Chakrabarti \& Fombonne, 2004). Obsessivecompulsive disorder has been found to be more frequent in the relatives of individuals with autism (Bolton et al., 1998; Mikali, Chakrabarti \& Fombonne, 2004).

Schizophrenia was once commonly believed to be associated with ASD but some studies have shown no increase in the rate of schizophrenia in families affected by ASD (Bolton et al., 1998). Ghaziuddin (2005) studied the family history of 97 individuals with either AS or high-functioning-autism and found $14 \%$ had a family history of 
schizophrenia. Interestingly, when comparing the family history of 58 individuals with AS with 39 individuals with high-functioning-autism (having normal IQ) significant differences were not detected but, subjects with AS were more likely to have relatives with schizophrenia (15.5\%) and depression (60.3\%) than were subjects with high functioning autism (Ghaziuddin, 2005). These findings may be suggestive of differences in risk factors for different diagnostic categories of ASD.

Researchers have a continued interest in assessing the possible relationships between ASD and other medical and psychiatric disorders. Although many studies have found other disorders to be related to ASD cases, no differences in familial history of medical and psychiatric disorders were found when compared to other developmental disabilities in one study (Williams, Oliver, Allard \& Sears, 2003). Most studies have involved relatively small samples of individuals with ASD and samples are often geographically restricted. In the current study, I explored the rate at which psychiatric disorders were observed in a large sample and compared these findings with previous research. Differences in the rate of related disorders in relatives across diagnostic categories were also explored to extend the current body of literature.

Parental Age

It seems clear that advanced parental age poses an increased risk for ASD, however, there are conflicting findings as to whether maternal or paternal age is the primary source of risk. Advanced maternal age (30-35+ years) has been implicated as a risk factor for ASD for more than 20 years (Tsai \& Stewart, 1983). In a Californian study, women over 35 were found to be three times more likely to have a child with autism compared to those under 20 (Croen, Grether \& Selvin, 2002). Maimburg and Vaeth (2006) 
also reported increased risk of ASD when mothers were over 35 years old. Evidence suggests that the older a mother is, the greater the risk of having a child with ASD, possibly due to the increased risk of birth complications (Glasson, Bower, Petterson, de Klerk, Chaney \& Hallmayer, 2004). Maternal age has been found to be an independent risk factor after controlling for other variables including paternal age (Glasson et al., 2004).

Results on maternal age as a risk factor have not been consistent, however, in a North Dakota sample of 78 autistic individuals, neither maternal, nor paternal ages were significantly different compared to the control group (Klug, Burd, Kerbeshian, Benz \& Martsolf, 2003). Similar findings of no significant differences between maternal age of cases and control groups were reported in an Irish sample of 49 autistic individuals (Cryan, Byrne, O'Donovan \& O'Callaghan, 1996) and a Japanese sample of 18 autistic NICU survivors compared with children with cerebral palsy and controls (Matsuishi, 1999). Williams, Oliver, Allard and Sears (2003) compared 102 autistic children with 106 children with non-autistic, developmental disabilities and found no significant difference in maternal (mean 26.3 years for autistic subjects) or paternal age (mean 28.9 years for autistic subjects).

Given the inconsistent results for maternal age, interest in paternal age has increased in recent years. In a Danish study of familial risk factors, the risk for autism increased for fathers 35 years or older compared to those 25-29 years at the time of the child's birth (Lauritsen, Pedersen \& Mortensen, 2005). Maternal age was a significant factor in this study but had no significant influence once the model was adjusted for paternal age, suggesting that maternal age is a proxy variable for paternal age (Lauritsen, 
Pedersen \& Mortensen, 2005). This finding contradicts an earlier study which found the opposite (Glasson, Bower, Petterson, de Klerk, Chaney \& Hallmayer, 2004).

The mixed findings on parental age led Reichenberg et al. (2006) to specifically investigate paternal age as a risk factor for ASD. Paternal and maternal age of 110 cases were considered and after adjustment for year of birth, socioeconomic status and maternal age, there was a significant increase in risk for ASD with advancing categories of paternal age (Reichenberg et al., 2006). Similar to previous studies, maternal age was significant only in the unadjusted models and was no longer significant after adjustment for paternal age (Reichenberg et al., 2006). In particular, fathers over the age of 40 were nearly 6 times more likely to have an offspring with ASD as compared to fathers under 30 years (Reichenberg et al., 2006). The increased risk for ASD and other developmental disabilities in older fathers is believed to be an indication of genetic mutations amongst the germline of older fathers (Reichenberg et al., 2006). The sample for this study consisted of only individuals diagnosed with autism and thus, the authors were not able to assess the differences between diagnostic categories including AS, AD and PDD-NOS. Another study was conducted and researchers were able to assess differences in parental age across two specific diagnostic categories and found that individuals with AS were more likely to be born outside of the optimal maternal age of 20-30 years than were individuals with high-functioning autism (Ghaziuddin, Shakal \& Tsai, 1995) suggesting that risk factors may vary depending on diagnostic category. This is one factor that the current study considers by comparing the average paternal and maternal age for each diagnostic category. 


\section{Social Class}

It has been repeatedly reported that autism is a disorder present across all socioeconomic classes (Cryan, Byrne, O’Donovan \& O'Callaghan, 1996; Fombonne, 2005a; Tidmarsh \& Volkmar, 2003). In a review of studies investigating autism and social class, studies conducted before 1980, and not after, found social class to be a risk factor for ASD and this has been interpreted as a referral bias related to limited access to services and inadequate case sampling methods prior to 1980 (Fombonne, 2005a). Although few researchers have examined social class systematically (Rutter, 2005), a variety of methods have been utilized to categorize social class. For example, Cryan, Byrne, O'Donovan and O'Callaghan (1996) found no significant difference in social group between the autistic group and controls using an Irish model of categorizing professional versus manual labour (O'Hare, Whelan, Commins, 1991). Most reviewers would agree that broadly defined social class has no association with ASD (Fombonne, 2005a; Rutter, 2005).

Social class has not been linked to ASD, however, maternal education has been isolated as a significant risk factor for the disorder in several studies. Klug et al. (2003) compared autistic subjects with normal controls found higher maternal education was a prenatal risk factor for autism whereas fathers' education and parent marital status were not. In a large Californian study, mothers with postgraduate education were twice as likely to have a child with ASD as compared to women with less than a high school education after adjustment for maternal age and other characteristics (Croen, Grether \& Selvin, 2002). In addition, when comparing autistic individuals to those with other developmental disabilities, mothers of children with autism were found to have 
significantly higher education (Akçakin, Polat \& Kerimoglu, 1993; Williams, Oliver, Allard \& Sears, 2003).

\section{Country}

Autism is known to occur across all cultural, racial and ethnic groups (Dyches, Wilder \& Obiakor, 2001). However, several studies have reported differing risk for ASD dependent on country of origin and immigrant status. In a study of familial risk factors, maternal place of birth was important as mothers born outside of Europe were at greater risk than those born in Denmark (Lauritsen, Pedersen \& Mortensen, 2004). In a second Danish study, foreign citizenship was also associated with increased risk of autism (Maimburg \& Vaeth, 2006). Similar findings linking autism to immigrant status and/or particular racial or ethnic groups have been reported in the UK (Goodman \& Richards, 1995; Wing, 1980), Sweden (Gillberg \& Gillberg, 1996) and California (Croen, Grether \& Selvin, 2002) yet findings were often contradictory based on small samples, or the studies lacked scientific rigour (Fombonne, 2003, 2005a; Rutter, 2005). In the current study, the large and diverse sample offered the opportunity to compare the existence of various risk factors across geographical regions and to gain a broader perspective on the occurrence of these experiences.

\section{Gender}

Boys are at greater risk for ASD than are girls (Volkmar \& Pauls, 2003). In a review of 32 studies between 1966 and 2004, not a single study identified more girls than boys having autism (Fombonne, 2005b). The mean ratio of boys:girls is 4.3:1 (Fombonne, $2005 \mathrm{~b}$ ) and the predominance amongst males is even higher for higher-functioning individuals with an estimate of 5.8:1 (Tidmarsh \& Volkmar, 2003). Whether risk factors 
vary depending on gender and diagnostic category were analyzed and reported in the current study.

\section{Birth Order}

Study results have been generally consistent in reporting that ASD is related to birth order. For example, first born children have been reported to be at increased risk for this disorder (Croen, Grether \& Selvin, 2002; Glasson et al., 2004). However, some studies have reported that size of the family is important in that firstborns are at increased risk for ASD in sibships of two and lastborn children are at greater risk in sibships of three or larger (Tsai \& Stewart, 1983; as reported in Rutter, 2005). The difference in risk based on birth order and size of family was supported and found to be unique to an autistic group when compared to a non-autistic, developmentally disabled control group (Williams, Oliver, Allard \& Sears, 2003). Researchers and clinicians have explained these findings in terms of stoppage rules in which parents' decisions to have further children are predictably affected by having a severely affected child (Jones \& Szatmari, 1988; Williams, Oliver, Allard \& Sears, 2003). Given the spectrum of severity in this disorder, it is plausible that parents apply these stoppage rules differently depending on severity as indicated by diagnostic category. For example, children with Asperger's Disorder may be older when their symptoms are recognized and they are diagnosed and given that their symptoms are likely to be less severe than those with autism, parents may have already gone on to have more children. In contrast, parents who had a severely affected child with Autistic Disorder who was identified early may be less likely to have more children. In the present study, it was anticipated that birth order would vary depending on diagnostic category. 


\section{Environmental Risk Factors}

The evidence from twin studies emphasizes the importance of genetic influences on the development of ASD yet also points to the possible importance of environmental risk factors. Monozygotic twins are virtually identical in genetic background and yet the expression of ASD and related characteristics are variable, which strongly suggests the possibility of environmental influence on the expression of the disorder (Rodier \& Hyman, 1998). Investigation of these environmental influences have identified a few concrete risk factors believed to be causative in a small number of cases, including congenital rubella, postnatal herpes encephalitis, gestational exposure to valproic acid and thalidomide (Rodier \& Hyman, 1998). The teratogenic effects of these diseases and drugs is believed to cause brain damage that may directly relate to the characteristics of the disorder or possibly to act as a trigger of the genetic susceptibility in individuals (Rodier \& Hyman,

1998). Although the identification of environmental factors through retroactive studies of pre- and perinatal histories is rare (Rodier \& Hyman, 1998) every clue as to the nature of the disorder is important and helpful in developing further hypotheses.

\section{Obstetric Complications}

Researchers have considered the experiences of mother and child in the pre and perinatal period in a number of studies to assess risk for ASD. Studies of obstetric complications have been particularly revealing in that children with ASD have been exposed to more complications during pregnancy and delivery than unaffected controls. (Akçakin, Polat \& Kerimoglu, 1993; Glasson et al., 2004; Li et al, 2002). For example, mothers of children with ASD have been found to be more likely to experience labour induction, threatened abortion, epidural caudal anesthesia and brief labour (Glasson et al., 
2004). Furthermore, the newborns later diagnosed with ASD were more likely to have experienced fetal distress, have had an Apgar score under 6 at 1 minute and have been delivered via cesarean section (Glasson et al., 2004). Increased frequency of low birth weight and birth defects in ASD cases have also been reported (Klug, Burd, Kerbeshian, Benz \& Martsolf, 2003; Maimburg \& Vaeth, 2006). In a study of neonatal intensive care unit (NICU) survivors, the prevalence of ASD cases was significantly higher than in the general population and meconium aspiration syndrome was more common in these ASD cases compared with the unaffected survivors of the NICU (Matsuishi, 1999). It is important to note that not all studies have found evidence to support increased obstetric adversity. An Irish sample comparing groups on low birth weight, gestational age, previous maternal loss of fetus and other factors, found no evidence that these obstetric complications were related to the development of ASD (Cryan, Byrne, O'Donovan \& O'Callaghan, 1996). Although no single obstetric complication has been directly linked to the development of ASD, the increased prevalence of complications commonly reported is worthy of ongoing investigation and may lead to the identification of causal pathways.

The pre- and perinatal experiences may differ in important ways for individuals with AD, PDD-NOS and AS. In one study, types of complications were similar but differed in frequency and severity depending on diagnostic category in that individuals with $\mathrm{AD}$ had increased birth complications compared to those with PDD-NOS who in turn, had more than those with AS (Glasson et al., 2004). Eaton et al. (2001) compared the obstetric histories of seven diagnostic groups and found that the autistic group had no significant differences compared to other disability groups, however, the Asperger's group were more likely to have had low birth weight and been slow to grow compared to other 
groups. In a study of 100 males with AS, cases were found to have experienced high rates of prenatal problems including severe prenatal infections, second trimester bleeding and preeclampsia and nearly $60 \%$ of cases had some perinatal complication although they were rarely severe (Cederlund \& Gillberg, 2004). Combined genetic and pre or perinatal factors were common in at least $11 \%$ and pre and perinatal factors were present in a total of $25 \%$ of AS cases (Gillberg \& Cederlund, 2005). In a small study comparing an AS group $(n=10)$ with a high-functioning autism group $(n=10)$ no significant differences in obstetric optimality were found (Ghaziuddin, Shakal \& Tsai, 1995). Given the debate that AS and high-functioning autism are not diagnostically distinguishable (Klin, McPartland \& Volkmar, 2005), this finding is not necessarily surprising. Mixed findings and limited studies comparing ASD categories necessitate the continued exploration of possible differences in genetic and/or environmental risk factors for each diagnostic category on the autistic spectrum.

Increased obstetric complications in ASD cases are generally not interpreted as direct causes of the disorder, but rather as symptoms of underlying genetic factors (Bolton et al., 1997; Gillberg \& Gillberg, 1983; Zwaigenbaum et al., 2002) or as an interaction between genetic and environmental factors resulting in a compromised prenatal experience (Glasson et al., 2004; Maimburg \& Vaeth, 2006; Rodier \& Hyman, 1998). Studies looking at pre and perinatal factors related to ASD have typically used sample sizes between 53-87 cases and the largest sample to date included 465 cases (Glasson et al., 2004). Given the potential importance of identifying the environmental risk factors related to the pre and perinatal experiences of children who develop ASD, it would be valuable to identify how common these factors are experienced. Based on the increased severity of 
symptoms in Autistic Disorder as compared to Asperger's Disorder, it was anticipated in the current study that a relationship between obstetric complications and diagnosis would exist.

\section{Severe Characteristics}

Individuals with ASD are known to exhibit severe behavioural excesses and deficits in functioning. Characteristics including self-injurious behaviour (SIB), repetitive self-stimulatory behaviour, sleep disturbances, lack of verbal communication and intellectual disability are commonly discussed in the literature but the specific prevalence of characteristics such as SIB and the related risk factors has not been extensively investigated (Baghdadli, Pascal, Grisi \& Aussilloux, 2003). Autistic individuals have been found to be significantly more likely to show self-injury, aggression and disruption to the environment as compared to people with intellectual disabilities (McClintock, Hall \& Oliver, 2003). In a recent parent report study comparing autistic subjects with individuals with a history of language impairment, behaviours such as atypical eating behaviour, abnormal sleep patterns, temper tantrums, and self-injurious behaviour were significantly more common in the children with autism (Dominick et al., 2007). The number of atypical behaviors was also related to the severity of social and communicative deficits as well as to restricted, repetitive behaviour but why is unclear (Dominick et al., 2007). The prevalence of self-injurious behaviour has been reported in $10 \%$ of an institutionalized population for the mentally retarded (Schroeder, Schroeder, Smith \& Dalldorf, 1978) and $53 \%$ in one autistic sample (Baghdadli, Pascal, Grisi \& Aussilloux, 2003) and 30\% of another sample (Dominick et al., 2007). Over 67\% of ASD cases had some degree of sleep disturbance as reported by parents (Dominick et al., 2007). McClintock, Hall and Oliver 
(2003) have highlighted the need for more data on prevalence of challenging behaviours and therefore, the prevalence of such characteristics as reported by parents of individuals with ASDs was analyzed as part of the current study.

Little is also known about the specific risk factors for challenging behaviours and severe symptomology in the ASD population. Gender (males are at greater risk), low communication skill and degree of intellectual disability have commonly been reported as risk markers for challenging behaviours such as aggression and self-injury in the intellectually disabled population (reviewed by McClintock, Hall \& Oliver, 2003). There is also some evidence to suggest that risk factors such as obstetric complications are related to the severity of ASD symptoms (Glasson et al., 2004). AS is considered milder on the spectrum of severity of symptoms and as previously discussed, individuals with AS are more likely to experience obstetric complications than normal controls, but fewer than autism groups who by definition are more severely affected (Glasson et al., 2004). Likewise, previous research has shown a link between IQ and obstetric complications in that those individuals with higher IQ experienced fewer complications (Gillberg \& Gillberg, 1983). In a study of risk factors for SIB, significant relationships were found between SIB and the perinatal condition, higher speech delay, higher adaptive delay and higher degree of autism (Baghdadli, Pascal, Grisi \& Aussilloux, 2003). I explored who on the spectrum is most at risk for these severe symptoms and what possible genetic and environmental risk factors may be related to these symptoms. For example, are individuals who experienced obstetric complications at greater risk for self-injurious behaviour or sleep disturbances? Any information that points specifically to the variables 
related to risk for these severe behavioural characteristics will assist in directing future studies on this topic.

Overview of Present Study

The primary focus of the current study was to identify the prevalence of genetic and other risk factors in a large, diverse sample and explore differences based on specific diagnoses. A high prevalence of any specific factor will help to guide researchers in focusing on that area in future studies. The premise behind this study is that autism is unlikely to have one single etiological pathway, but rather multiple factors may be at play in the development of the disorder. It was anticipated that multiple factors, both genetic and environmental, would occur frequently in this sample. Furthermore, possible differences in risk factors based on the three diagnostic categories including AD, PDDNOS and AS were analyzed. Given the heterogeneity in the expression of characteristics on the spectrum and the range of severity in symptoms, it was anticipated that differences in the type and number of risk factors would be found depending on the diagnostic category. For example, it is likely that children with Autistic Disorder would have experienced more or different risk factors as compared to Asperger's Disorder given that these two diagnoses represent the extreme ends of the spectrum.

A secondary purpose to this study was to identify the prevalence of some key severe characteristics such as self-injurious behaviour, sleep problems, frequent selfstimulatory behaviour and non-verbal communication and to investigate if these had any relationship to various factors in the child's family history or pre or perinatal period. If risk factors for each specific disorder and severe behavioural characteristics can be 
positively identified, this could lead to the earlier identification and therefore earlier access to intervention for children and their families.

\section{Method}

\section{Survey Development}

The present study utilized archival data from an extensive, web-based survey that was designed to collect general information on autism from the parents of children with ASD. The survey was developed to explore a variety of issues and to gather general data on demographics, family history, diagnosis, child characteristics and treatment data. The survey was quite extensive with a total of 72 questions and many questions were openended to afford the opportunity for responses that may not have been anticipated by the researchers. Refer to Appendix A for a copy of the survey, excluding the treatment section. The treatment data from this survey has been analyzed in a separate study which found that type of treatment, number of treatments and discontinuation of treatments used, differed between groups based on diagnostic category (Hicks, Bowker \& Wells, 2006). In addition, there were significant differences in how children responded to treatments and suggested that individual types and severity of characteristics may impact on treatment outcome. While treatment data was not directly relevant to the current study, the previous significant findings between groups in this data set warranted further investigation.

Other surveys have directly asked parents about their beliefs on the cause(s) of ASD. One such study found parents reported that they believed genetics, perinatal factors, prenatal factors, diet and vaccines to be significant contributory factors (Mercer, Creighton, Holden \& Lewis, 2006). Although some of these factors are empirically well 
supported, others, such as diet have had inconclusive findings (Wing \& Potter, 2002) and in the case of vaccines, have repeatedly not been substantiated in the literature (Tidmarsh \& Volkmar, 2003). Further parent misperceptions were noted including an inaccurately high estimate of recurrence risk for the disorder (Mercer, Creighton, Holden \& Lewis, 2006). Given the tendency for misperceptions and inaccuracies in parent beliefs about the etiology of ASD, one of the strengths of the current study is that the survey questions did not ask parents for their beliefs, but rather, asked parents to report particular pieces of information about their child and family which could then be analyzed as associated factors. A possible limitation to the current study was the dependency on the accuracy of parent recall of information.

\section{Survey Distribution}

The survey was posted online for three months in the fall of 2003 on autism-related websites and on autism-related email distribution lists in Canada and the United States. A total of 1,034 parents completed the survey online. After all data had been collected it was exported into a statistical software package (SPSS $\circledast$ ) for data analysis.

\section{Results}

\section{Participants' Diagnoses}

The response to this survey included 1034 completed surveys. As part of the survey, respondents were asked to indicate their child's diagnosis as PDD-NOS, Autistic Disorder, Asperger's disorder or Other. Those who reported a diagnosis other than an ASD or did not respond to this question $(n=65)$ were eliminated from the dataset which left 969 subjects. Respondents were also asked to indicate if their child's diagnosis had 
changed at any point after the initial diagnosis. Of these 969 survey respondents, 196 (20.2\%) indicated that "yes" their child's diagnosis had changed. The most current diagnosis of the child was presumed to be the most accurate and was chosen as the most relevant for all analyses in this study and therefore, any surveys that indicated a current diagnosis other than an ASD were eliminated $(n=51)$ which resulted in a final pool of 918 participants. The majority of this sample had a diagnosis of Autistic Disorder ( $n=496$; $54 \%)$, followed by PDD-NOS $(n=278 ; 30.3 \%)$ and Asperger's $(n=144 ; 15.7 \%)$. The diagnosis of ASD was based on the use of two or more assessment techniques or tools in $72.4 \%(n=482)$ of cases with $26.4 \%(n=176)$ of remaining cases based on only one assessment technique. The number of hours of clinical observation to make a diagnosis for the individual was reported in categories and it was most common $(78 \%, n=693)$ for individuals with ASD to be diagnosed after five or fewer hours of observation. Based on chi-square analysis, there is a significant relationship between diagnosis and number of hours of observation $\chi 2(8, N=888)=32.771, p<.001$. In particular, individuals with Autistic Disorder, who are at the severe end of the ASD spectrum, were more likely than expected $(f=389, f e=378.5)$ to have been observed for five or fewer hours and less likely than expected to have been observed for twenty-one or more hours $(f=25, f e=31.7)$. The reverse was true for the Asperger's group, who are at the least severe end of the spectrum. This group were less likely to be diagnosed in five or fewer hours than expected $(f=85, f e$ $=106.1)$ and more likely than expected to be observed for twenty-one or more hours $(f=$ $21, f e=8.9$ ). The results of this comparison are reported in Appendix B.

Given that one-fifth ( $n=196)$ of the sample $(N=969$; before elimination of "other" diagnoses) indicated the child's diagnosis had changed at some point, it seemed 
appropriate to investigate the nature of that change. The severity of the disorder is by definition, a spectrum, so the nature of the change was determined by whether the child's new diagnosis was now more severe (ex. was Asperger's and is now PDD-NOS) or less severe (ex. was Autistic Disorder and is now PDD-NOS) on the spectrum. The results show that more than a third of those who had a change in diagnosis went from a less severe diagnosis to a diagnosis that was more severe on the autism spectrum but almost equally as many had the reverse and went from being more severe on the spectrum to less severe (see Table 2). Also, a quarter of the participants who were originally on the spectrum but had a change in diagnosis at some point were no longer on the spectrum (see Table 2). Those who were no longer diagnosed with an ASD were eliminated from all further analyses. 
Table 1

Participants' Diagnoses

\begin{tabular}{lcc}
\multicolumn{1}{c}{ Diagnosis } & $n$ & Percentage \\
\hline Asperger's Disorder & 144 & $15.7 \%$ \\
PDD-NOS & 278 & $30.3 \%$ \\
Autistic Disorder & 496 & $54.0 \%$ \\
Total & 918 & $100 \%$ \\
\hline
\end{tabular}


Table 2

Nature of Change of Diagnosis

Change

$n$

Percentage

From less to more severe on spectrum

71

$36.2 \%$

From more to less severe on spectrum

55

$28.1 \%$

An ASD to "other"

50

$25.5 \%$

"other" to an ASD

6

$3.1 \%$

ASD to undisclosed diagnosis

14

$7.1 \%$

Total

196

$100 \%$ 


\section{Social Class}

Survey respondents were asked to indicate their family income by category and to provide the occupation of both parents. A summary of family income is provided in Table 3. The vast majority of this sample had an average annual family income over $\$ 40,000$ $(72.1 \%, n=613)$ with $30.5 \%(n=259)$ of the sample earning over $\$ 80,000$ per year. There was no significant difference in income across diagnostic groups $\chi 2(8, N=850)=11.905, p$ $=.155$.

The occupation of each parent was coded based primarily on the National Occupational Classification structure (HRDS, 2006) and is reported in Table 4. The interrater reliability on the coding of mothers' occupations was $89.1 \%$ and it was $81.9 \%$ for fathers' occupations based on comparison of $25 \%$ of cases by an independent coder. Mothers most commonly stayed at home to take care of children (38.4\%) followed by employment in Social Science jobs such as education or government (16.8\%). Fathers were most often employed in Natural or Applied Sciences (15.3\%) that includes engineering and high technology industry, or Social Science positions (14.7\%). 
Risk Factors in ASD 32

Table 3

Family Average Annual Income

\begin{tabular}{lcc}
\hline & $n$ & Percentage \\
Under $\$ 20,000$ & 70 & 8.2 \\
\cline { 2 - 3 }$\$ 20,000-\$ 40,000$ & 167 & 19.6 \\
$\$ 40,000-\$ 60,000$ & 182 & 21.4 \\
$\$ 60,000-\$ 80,000$ & 172 & 20.2 \\
Above $\$ 80,000$ & 259 & 30.5 \\
Total & 850 & 100 \\
\hline
\end{tabular}


Table 4

Occupation of Parents

Management

Business

Natural \& Applied Science

\begin{tabular}{cccc} 
Mothers & $\begin{array}{c}\text { Percentage of } \\
\text { Mothers }\end{array}$ & Fathers & $\begin{array}{c}\text { Percentage of } \\
\text { Fathers }\end{array}$ \\
\hline 19 & $2.1 \%$ & 96 & $11.1 \%$ \\
100 & $11.3 \%$ & 106 & $12.3 \%$ \\
35 & $3.9 \%$ & $132 * *$ & $15.3 \% * *$ \\
& & & \\
86 & $9.7 \%$ & 52 & $6.0 \%$ \\
149 & $16.8 \%$ & 127 & $14.7 \%$
\end{tabular}

Social Science, Education,

149

$16.8 \%$

127

$14.7 \%$

Government

Art, Culture, Recreation

41

$4.6 \%$

24

$2.8 \%$

Trades, Transportation or Equipment

$\begin{array}{llll}4 & 0.5 \% & 108 & 12.5 \%\end{array}$

Primary Industry

1

Sales, Service

49

$0.1 \%$

5

$0.6 \%$

Processing, Manufacturing

3

$5.5 \%$

$10.6 \%$

3

$0.3 \%$

$5.0 \%$

Homemaker

Student

$341^{*}$

$38.4 \% *$

$2.7 \%$

$0.7 \%$

Self-Employed

24

13

Unemployed

$1.5 \%$

$0.7 \%$

Total

$2.5 \%$

$4.6 \%$

887

$100 \%$

$3.0 \%$

$100 \%$

$*=$ most common occupation for mothers

** = most common occupation for fathers 


\section{Place of Residence}

Participants were asked where they currently reside and these responses were categorized into 5 broad categories as reported in Table 5. The majority of the sample were currently living in the United States $(n=707,78 \%)$ and when combined with Canada, $92.9 \%$ were living in North America. The remaining $7.1 \%(n=64)$ were living in countries around the globe. A chi-square analysis was attempted to compare place of residence with diagnostic group but numbers were too low outside of North America for the results to be reliable, $\chi 2(8, N=906)=26.68, p=.001$, with 4 cells $(26.7 \%)$ with an expected count less than five. There were no significant relationships between place of residence and any of the individual risk factors in this sample (see summary of results in Appendix C).

\section{Gender}

This sample of individuals with an ASD included $762(83 \%)$ males and $156(17 \%)$ females which represents a 4.9:1 ratio of males to females. There was no significant difference in the number of boys and girls in each diagnostic category, $\chi 2(2, N=$ $918)=0.99, p=.609$. Chi-square analyses were conducted to investigate if gender was related to any of the risk factors of interest. There was a significant relationship between gender and the presence of a comorbid medical condition, $\chi 2(1, N=821)=8.11, p<.01$. In particular, girls with ASD were more likely to have a comorbid medical condition than expected $(f=77, f e=61.8)$ while the opposite was true for boys with $\operatorname{ASD}(f=288, f e=$ 303.2). No other risk factors differed in relation to gender (see Appendix D). Gender differences occurred in two types of severe characteristics. First, girls with ASD 
Risk Factors in ASD 35

Table 5

Place of Residence

n Percentage

United States of America

707

$78.0 \%$

Canada

135

$14.9 \%$

Europe

33

$3.6 \%$

Australia or New Zealand

16

$1.8 \%$

Other

15

$1.7 \%$

Total

906

$100 \%$ 
were more likely than expected to have sleep disorders $(f=89, f e=75.5)$ as compared to boys $(f=348, f e=361.5)$. Second, while no gender differences existed in who is likely to engage in self-injurious behaviour, $\chi 2(1, N=906)=0.46, p=.496$, there was a significant relationship between gender and the severity of $\operatorname{SIB}, \chi 2(2, N=289)=9.76, p<.01$. Girls were over-represented in the moderate $(f=26, f e=23)$ and severe $(f=19, f e=13.1)$ categories of SIB while boys were more likely to display mild $\operatorname{SIB}(f=81, f e=72.2)$. Age

The age of the child with ASD was calculated as of the time the survey was completed and these results are reported in Table 6 . The sample primarily included children 12 years of age and younger $(n=792,86.5 \%)$. In Table 7 , the age at which the child with ASD was diagnosed is reported in categories. The range of ages at the time of the diagnosis of an ASD was from 1 to 29 years with a mean of 4.4 years $(S D=2.97)$. Most children were diagnosed in the preschool years of 5 years and younger and this was true for all three diagnostic groups (see Figure 1). Chi-square analysis revealed a significant relationship between diagnosis and age of diagnosis, $\chi 2(4, N=905)=20.51, p<$ .001. In Figure 1, it is apparent that children with Asperger's disorder were the least likely to be diagnosed in the preschool years and were more likely to receive their diagnosis in middle childhood or the teenage years compared to those with PDD-NOS or Autistic Disorder. 
Table 6

Age of Child with ASD at Time of Survey Completion

\begin{tabular}{lccc}
\hline & $n$ & Percentage & $\begin{array}{c}\text { Cumulative } \\
\text { Percentage }\end{array}$ \\
\cline { 2 - 4 }$\leq 5$ years & 378 & 41.3 & 41.3 \\
$6-12$ years & 414 & 45.2 & 86.5 \\
$13-18$ years & 92 & 10.1 & 96.6 \\
$>18$ years & 31 & 3.4 & 100 \\
\hline
\end{tabular}


Table 7

Age of child with ASD at Time of Diagnosis

\begin{tabular}{lccc}
\hline & $n$ & Percentage & $\begin{array}{c}\text { Cumulative } \\
\text { Percentage }\end{array}$ \\
\cline { 2 - 4 }$\leq 5$ years & 689 & 76.0 & 76.0 \\
$6-12$ years & 197 & 21.7 & 97.7 \\
$13-18$ years & 19 & 2.1 & 99.8 \\
$>18$ years & 2 & 0.2 & 100 \\
\hline
\end{tabular}


Figure 1

Percent of Group Diagnosed at Specified Age

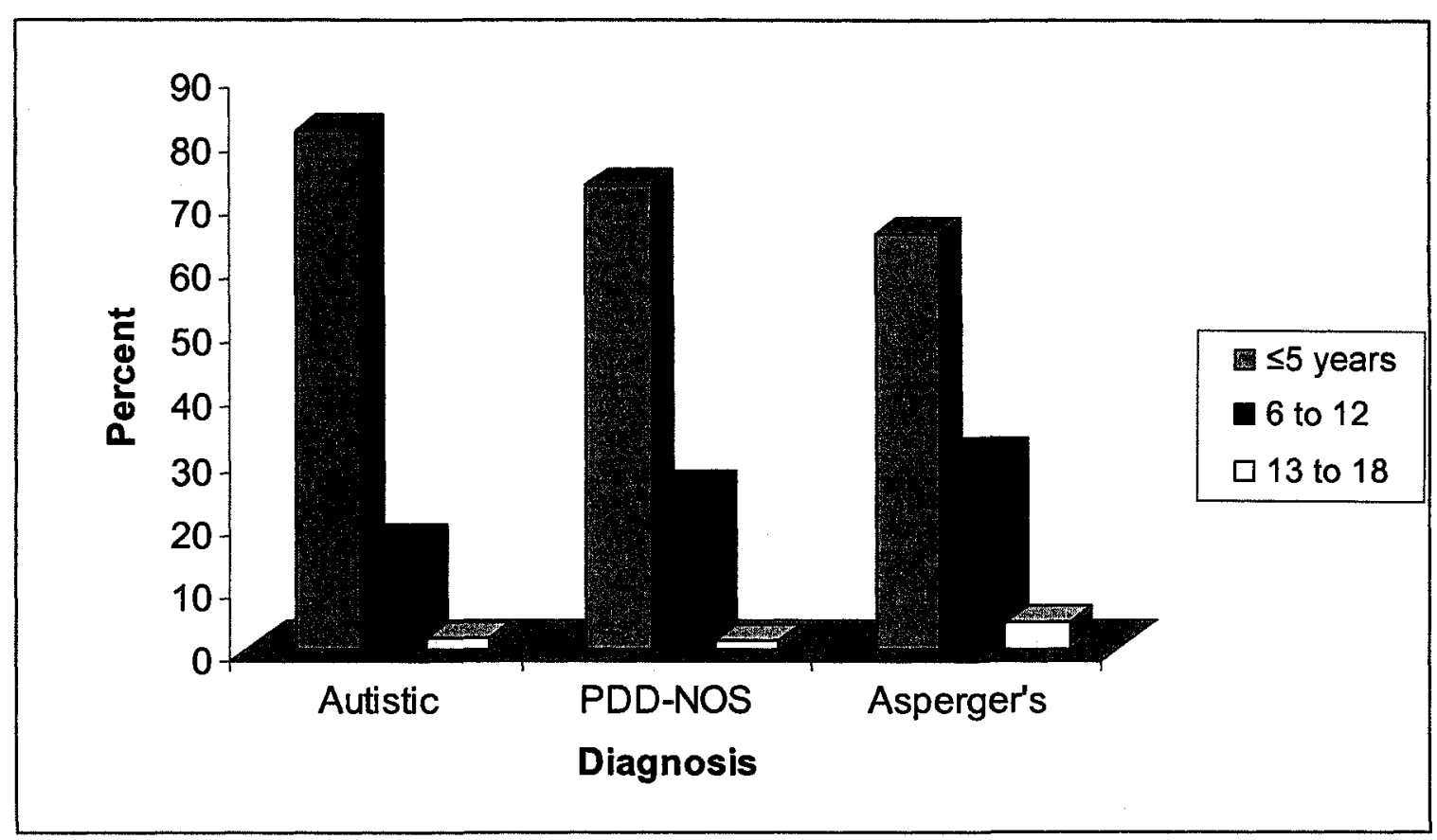




\section{Associated Medical Conditions}

Participants were asked to indicate whether the individual with ASD had a comorbid medical condition and if so, to report the type of medical condition(s). Of the 821 participants that answered these questions, $39.8 \%(n=365)$ indicated the presence of one or more medical conditions. It was apparent in the dataset that some respondents had indicated that "yes" a comorbid medical condition was present but did not indicate what condition and the reverse also happened where the respondent answered "no" to other medical conditions but listed a condition anyway. This is reflected in a slight discrepancy in total number of responses, in that 368 participants listed a comorbid type of medical condition. A chi-square analysis that compared the number of cases that indicated the presence of a comorbid medical condition across the three diagnostic categories revealed no significant differences between groups, $\chi 2(2, N=821)=4.52, p=.104$. This indicated that individuals with any of the ASD diagnoses are equally likely to have a comorbid medical condition.

The qualitative responses for type of medical condition were coded into five major categories including: (1) “Chromosomal Disorders” such as Down's Syndrome, Tuberous Sclerosis, Fragile X, Neurofibromotosis, Klinefelter's Syndrome, etc.; (2) "Neurological Conditions" such as epilepsy, cerebral palsy, hydrocephalus, spina bifida, etc.; (3) "Major Systems" such as organ disease or damage, diabetes, physical deformity, vision loss, hearing loss, etc.; (4) "Mental Health" such as mood or psychiatric diagnoses; ADHD, OCD; depression, anxiety, etc., and, (5) "Other" conditions including recurrent ear infections, allergies, eczema, etc. For cases in which multiple conditions were listed, the most significant condition was used for purposes of categorization in order of 
chromosomal, neurological, major systems, mental health and other. For example, if a child had Fragile X, seizures and allergies, they would only be reported in the "chromosomal" category. Inter-rater reliability for this coding scheme was assessed for $30 \%(n=114)$ of cases and was calculated at $95.6 \%$ agreement. The results for the types of medical conditions by category are reported in Table 8 . The "other" category accounted for the largest proportion of cases, $(17.3 \% ; n=159)$. Chi-square analysis comparing type of medical condition and diagnostic category, found significant differences, $\chi 2(8, N=368)=27.91, p<.001$, as reported in Table 9. The "other" category was the most common type of medical condition for each diagnosis. However, the second most common type of medical condition differed based on diagnosis. Neurological conditions were the second most common type of condition for those individuals with PDD-NOS (23.9\%, $\mathrm{n}=27)$. In the Asperger's and Autistic Disorder cases, "major systems" were the second most commonly reported type of comorbid condition with $29.2 \%$ of each category represented. The Autistic Disorder group was most likely to report neurological conditions $(54.5 \%, n=42)$, disorders of major systems $(46.3 \%, n=37)$ and "other" conditions $(55.3 \%, n=88)$. Mental health conditions were reported most often in the Asperger's group $(44.4 \%, n=16)$ whereas chromosomal disorders were highest for the PDD-NOS group $(50.0 \%, n=8)$. 
Risk Factors in ASD 42

Table 8

Type of Medical Condition Reported Across All Groups

$n \quad$ Percentage

Chromosomal

16

4.3

Neurological

77

20.9

Major Systems

80

21.7

Mental Health

36

9.8

Other

159

43.2

Total

368

100 
Table 9

Comparison of Comorbid Medical Condition Types by Diagnosis

Type of medical condition

\begin{tabular}{|c|c|c|c|c|c|c|}
\hline & & \multicolumn{5}{|c|}{ Type of medical condition } \\
\hline & & Chromosomal & Neurological & $\begin{array}{c}\text { Major } \\
\text { Systems }\end{array}$ & $\begin{array}{l}\text { Mental } \\
\text { Health }\end{array}$ & Other \\
\hline \multirow{4}{*}{ PDD-NOS } & Count & 8 & 27 & 22 & 11 & 45 \\
\hline & Expected & 4.9 & 23.6 & 24.6 & 11.1 & 48.8 \\
\hline & $\begin{array}{l}\% \text { within } \\
\text { diagnosis }\end{array}$ & 7.1 & 23.9 & 19.5 & 9.7 & 39.8 \\
\hline & $\begin{array}{l}\% \text { within } \\
\text { condition }\end{array}$ & 50.0 & 35.1 & 27.5 & 30.6 & 28.3 \\
\hline \multirow{4}{*}{$\begin{array}{l}\text { Autistic } \\
\text { Disorder }\end{array}$} & Count & 7 & 42 & 37 & 9 & 88 \\
\hline & Expected & 8.0 & 38.3 & 39.8 & 17.9 & 79.1 \\
\hline & $\begin{array}{l}\% \text { within } \\
\text { diagnosis }\end{array}$ & 3.8 & 23 & 29.2 & 4.9 & 48.1 \\
\hline & $\begin{array}{l}\% \text { within } \\
\text { condition }\end{array}$ & 43.8 & 54.5 & 46.3 & 25.0 & 55.3 \\
\hline \multirow{4}{*}{$\begin{array}{l}\text { Asperger's } \\
\text { Disorder }\end{array}$} & Count & 1 & 8 & 21 & 16 & 26 \\
\hline & Expected & 3.1 & 15.1 & 15.7 & 7.0 & 31.1 \\
\hline & $\begin{array}{l}\% \text { within } \\
\text { diagnosis }\end{array}$ & 1.4 & 11.1 & 29.2 & 22.2 & 36.1 \\
\hline & $\begin{array}{l}\% \text { within } \\
\text { condition }\end{array}$ & 6.3 & 10.4 & 26.3 & 44.4 & 16.4 \\
\hline
\end{tabular}


Cases from all categories seemed to include descriptions of gastro-intestinal problems. Further coding was undertaken to categorize all cases, across all categories, into a dichotomous variable for presence or absence of any gastro-intestinal problems which included symptoms such as persistent diarrhea, constipation, "leaky gut", dietary intolerances, irritable bowel syndrome, reflux and similar descriptions. The inter-rater agreement was $99.1 \%$ for this coding scheme based on comparison of $30 \%$ of the cases. Of the 368 respondents who indicated some form of comorbid medical condition, 94 (25\%) listed a gastro-intestinal symptom which represents $10.2 \%$ of the ASD cases overall. The three diagnostic groups were equally likely to have reported gastro-intestinal problems, $\chi 2(2, N=368)=3.05, p=.218$.

\section{Other Cases of ASD in Family}

Participants were asked to report if other cases of ASD were present in the family. A total of 891 (97\% of sample) responded to this question and $242(27.2 \%)$ indicated that there was at least one other case of ASD in their family and therefore, 649 (72.8\%) did not have other identified cases of ASD in the family. Participants were asked to indicate what relation the individual(s) with ASD are to the son or daughter reported on in the survey. From this data, a total number of cases of ASD per family were calculated. As reported in Table 10, of the families who had at least one other case of ASD in addition to the child reported on in the survey, the majority had only one other case in the family $(91.3 \%$, $n=221$ ). Other cases of ASD were reported to be first degree relatives in $37.3 \%$ of families and second degree relatives in $57 \%$ of families while only $5.4 \%$ reported both first and second degree relations with an ASD. Furthermore, a significant difference existed between diagnostic groups in terms of who was more likely to have other cases of 
ASD in the family, $\chi 2(2, N=891)=10.53, p<.01$. Specifically, individuals with PDD-NOS were less likely than expected to have a positive family history $(n=58, f e=72.8)$ and the Asperger's group was more likely to have a family history of $\operatorname{ASD}(n=51, f e=37.8)$. Approximately a quarter of the PDD-NOS (21.6\%) and Autistic Disorder groups (27.5\%) each reported having at least one other case of ASD in the family while the Asperger's Disorder group was reported to include more families affected by other cases $(36.7 \%)$. In other words, 1 in 4 families who have a child with Autistic Disorder or PDD-NOS have at least one other case of ASD in the family. In comparison, 1 in 3 families with a child with Asperger's Disorder have at least one other case of ASD in the family. 
Table 10

Number of Other Cases of ASD in Family

\begin{tabular}{ccc}
\hline & $n$ & Percentage \\
\cline { 2 - 3 } 3 & 221 & 91.3 \\
3 & 8 & 3.3 \\
4 & 7 & 2.9 \\
5 & 5 & 2.1 \\
6 & 0 & 0.0 \\
7 & 0 & 0.0 \\
Total & 1 & 0.1 \\
\hline
\end{tabular}


Family History of Mental Illness

Participants were asked to indicate if there is a history of mental illness in their family. The response rate to this question was $94.3 \%(n=866)$ with $49.3 \%(n=427)$ of the responders positively indicating a history of mental illness in one or more family members. Chi-square analysis found significant differences between groups, $\chi 2(2, N=$ $866)=11.21, p<.05$. Specifically, of the 427 cases who had a history of mental illness in the family, $50.1 \%(n=214)$ were from the Autistic Disorder group and the remaining cases were split between the PDD-NOS group $(30.7 \%, n=131)$ and the Asperger's Disorder group $(19.2 \%, n=82)$. However, $62.1 \%$ of those with an Asperger's Disorder had a history of family mental illness and this represented a higher than expected proportion of this group $(f=82, f e=65.1$ ) whereas, the PDD-NOS group was equally split between those who had a family history (49.4\%) and those who did not (50.6\%). The Autistic Disorder groups were slightly less likely to have a family history $(f=214)$ than expected $(f e=231.3)$ with $45.6 \%$ of the group indicating a family history of mental illness. Responders also listed the type of the family members' disorder and their relationship to the child with ASD. From this information, the total number of relatives per family was calculated and is reported in Table 11. The type of mental illnesses reported often included depression, anxiety, bipolar disorder, schizophrenia, obsessive compulsive disorder and attention deficit hyperactivity disorder, for example. 
Table 11

Number of Relatives with History of Mental Illness

\begin{tabular}{ccc}
\hline & $n$ & percentage \\
\cline { 2 - 3 } 1 & 439 & 50.7 \\
2 & 151 & 17.4 \\
3 & 130 & 15.0 \\
4 & 73 & 8.4 \\
5 & 71 & 8.2 \\
6 & 1 & 0.1 \\
7 & 0 & 0.0 \\
Total & 1 & 0.1 \\
\hline
\end{tabular}




\section{Parental Age}

Birth dates for the child with ASD and both parents were requested in the survey. The age of each parent at the time of the birth of the child with ASD was calculated in years. Data was available for 888 mothers and 878 fathers. The age of mothers at the time of the birth of the child with ASD ranged from 15 to 46 years with a mean of 30.39 years $(S D=5.436)$. The age of fathers ranged from 18 years to 66 years with a mean of 32.58 years $(S D=6.113)$. The age of both parents were categorized into age groupings and are reported in Table 12. There was no significant difference in age of mother, $\chi 2(10, N=$ $888)=13.52, p=.196$, nor in age of father, $\chi 2(10, N=878)=15.37, p=.119$, across the three different diagnostic groups. 


\section{Table 12}

Age of Parents at Time of Child's Birth

\begin{tabular}{ccccc}
\hline & $\begin{array}{c}\text { Number of } \\
\text { Mothers }\end{array}$ & $\begin{array}{c}\text { Percentage of } \\
\text { Mothers }\end{array}$ & $\begin{array}{c}\text { Number of } \\
\text { Fathers }\end{array}$ & $\begin{array}{c}\text { Percentage of } \\
\text { Fathers }\end{array}$ \\
\cline { 2 - 5 } $20-24$ & 13 & 1.5 & 3 & 0.3 \\
$25-29$ & 117 & 13.2 & 73 & 8.3 \\
$30-34$ & 260 & 29.3 & 195 & 22.2 \\
$35-39$ & 287 & 32.3 & 305 & 34.7 \\
40 or older & 169 & 19.0 & 208 & 23.7 \\
Total & 42 & 4.7 & 94 & 10.7 \\
\hline
\end{tabular}




\section{Birth Order and Family Size}

The number of children in each family and their ages was reported in the survey. Analyses revealed that $90 \%$ of families had 3 or fewer children including the child with ASD as reported in Table 13. The size of families did not differ significantly based on the specific diagnosis of the child with ASD, $\chi 2(10, N=918)=5.98, p=.817$. The children with ASD were the only child in the family in $21.5 \%$ of cases.

From the birthdates of the children in each family, the birth order of the child with ASD was determined and the full table of results is available in Appendix B. A significant relationship was found between diagnostic groups and birth order, $\chi 2(6, N=904)=22.10, p$ $\leq .001$. The majority of individuals with an ASD were either first born or only children $(50.6 \%, n=457)$ and this holds true for each diagnostic category as seen in Figure 2. However, the Asperger's group were particularly likely to be first born or only children with $65.5 \%$ in this grouping which was more than expected $(f=93, f e=71.8)$. There also appears to be a trend towards children with Autistic Disorder being less likely to be first born and more likely to be later born than would be expected (refer to Appendix E). 
Risk Factors in ASD 52

Table 13

Number of Children in Family

1

197

$n$

Percentage

2

403

43.9

3

226

24.6

4

65

7.1

5

23

2.5

$>5$

4

0.4

Total

918

100

4
4


Figure 2

Birth Order within Diagnostic Groups

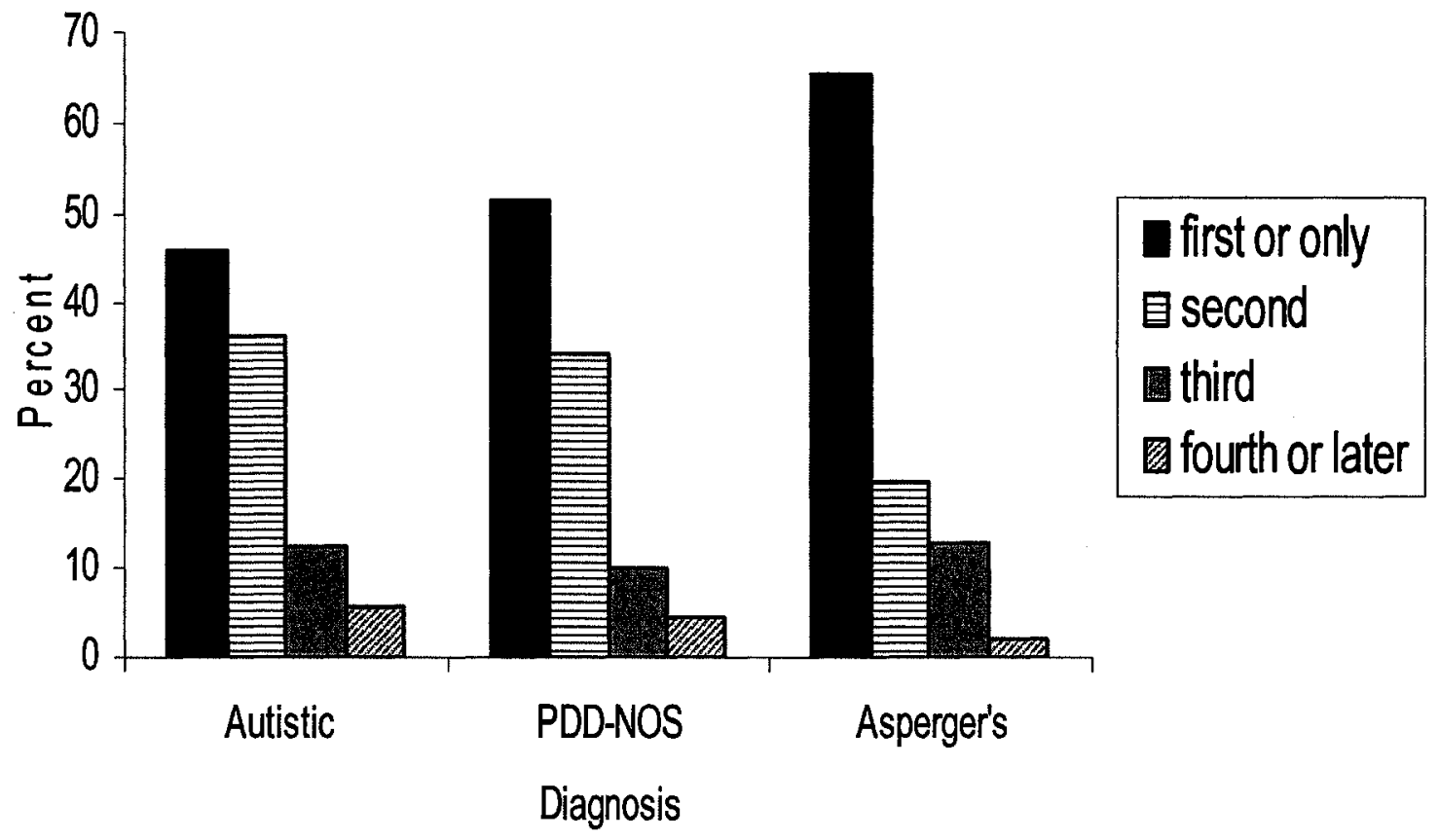




\section{Obstetric Complications}

According to the data, $51.8 \%(n=470)$ of individuals with an ASD experienced one or more birth complications. The complications are broken down into four categories as shown in Figure 3 and the inter-coder reliability was $83.9 \%$ based on independent coding of $25 \%$ of cases. "Maternal health" included serious illness, accident and use of medications during pregnancy; "labour/delivery complications" included breech birth, assisted delivery, problems of the placenta, cord problems, etc. and "neonatal health" included NICU admission, hydrocephalus, respiratory distress, jaundice, etc. Individuals who reported only one type of complication were categorized as maternal health problems at time of birth, labour or delivery problems or neonatal health problems. Any case that indicated complications across two or more categories was identified as having multiple types of complications. There was no significant difference in the presence of obstetric complication, $\chi 2(2, N=908)=4.14, p=.126$, nor in the type of obstetric complication, $\chi 2(6, N=458)=5.11, p=.530$, across diagnostic groups.

The gestational age of the child with ASD at the time of birth was categorized as preterm, full-term and post mature. The vast majority were born full-term with $73.2 \%$ of the sample being born between 37 and 41 weeks of gestation. Infants born before 37 weeks made up $17.5 \%$ of the sample and $9.3 \%$ were born at 42 weeks or later. A relationship exists between diagnosis and gestational age at delivery, $\chi 2(4, N=$ $824)=12.79, p \leq .01$, as seen in Figure 4. In particular, the Asperger's group was less likely to have been born premature than expected $(f=15, f e=22.4)$ and were almost twice as likely to be overdue than expected $(f=21, f e=12.0)$. However, the Autistic Disorder group was more likely to have been born premature than was expected $(f=87, f e=77.9)$. 
Figure 3

Categories of Obstetric Complications for all Individuals with ASD

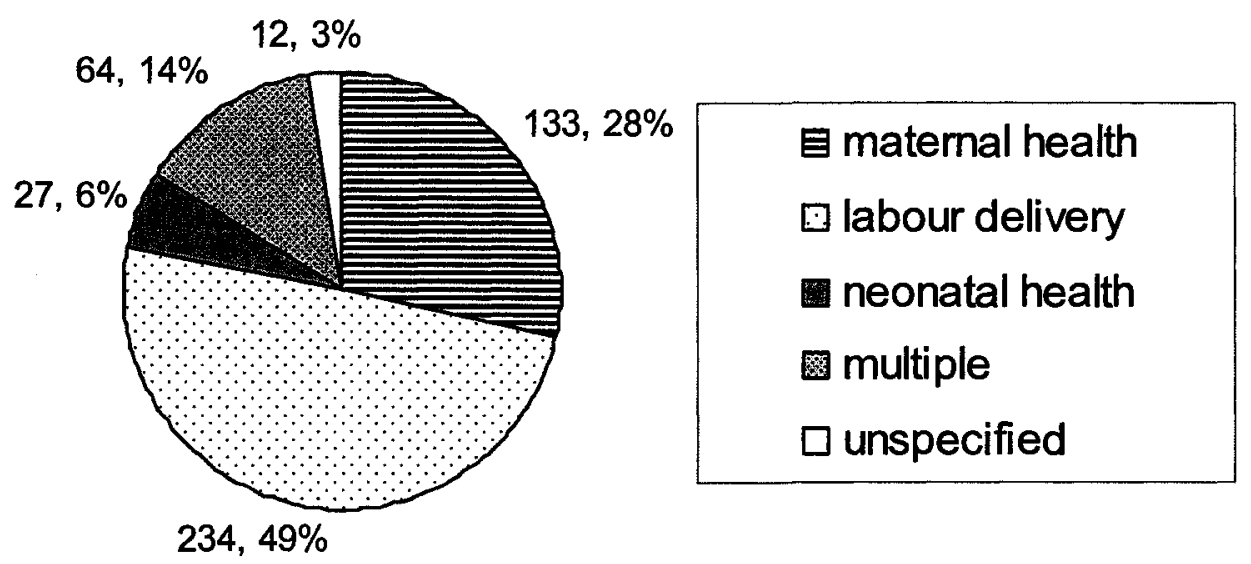


Figure 4

Gestational Age at Birth in Weeks

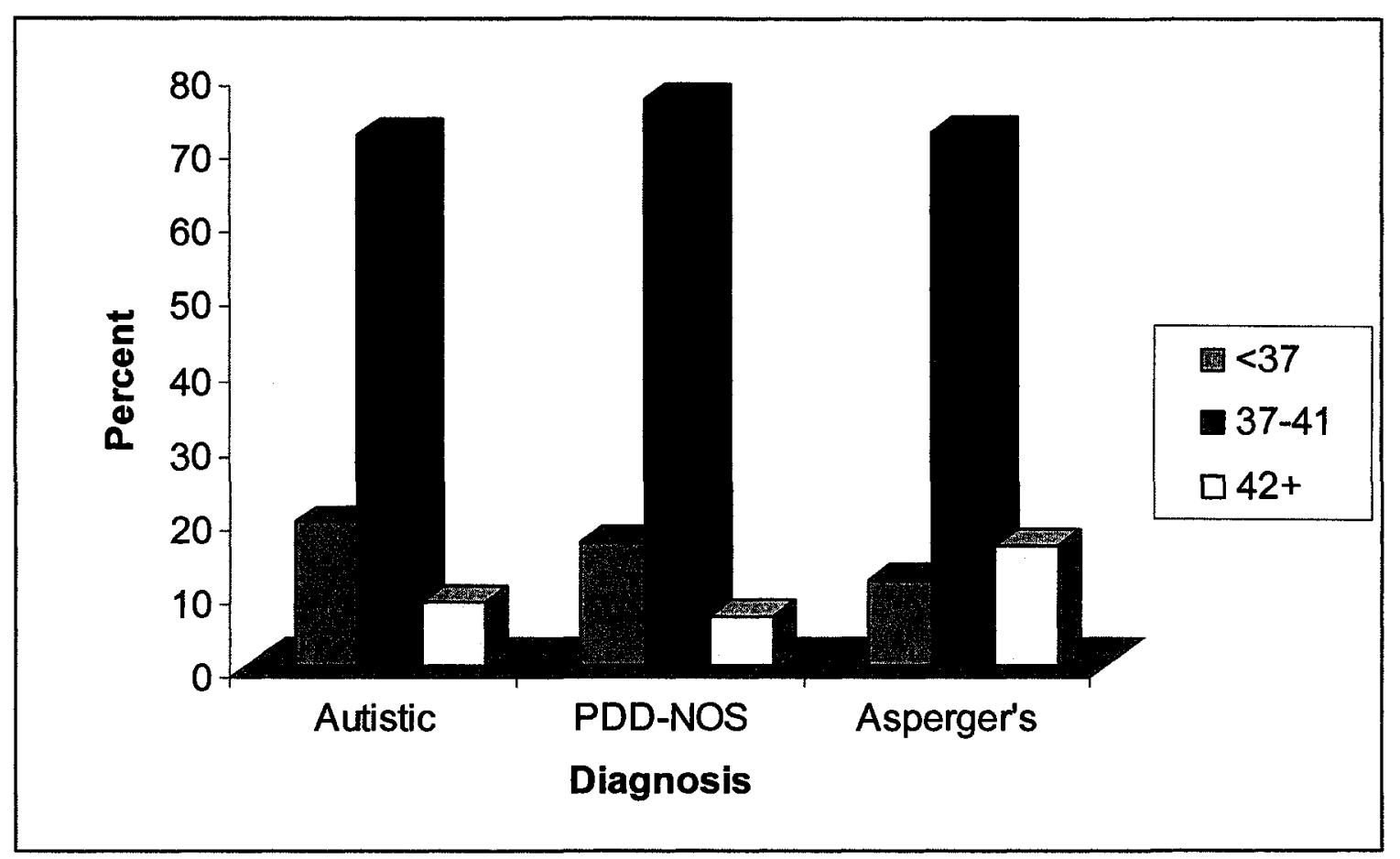


Number of Risk Factors Related to Each Disorder

Six key risk factors were combined to create an aggregate score of total number of risk factors experienced by each child with ASD. Participants received one point towards a total risk score if they had a father over the age of 35 at time of their birth, mother over the age of 35 at time of their birth, at least one birth complication, at least one comorbid medical condition and a point for each additional case of ASD in the family and a point for each additional family member with a mental health disorder. Total risk scores ranged from 0 to 11 with a mean of 2.76 risk factors $(S D=1.951)$ across all participants. Further analysis of the mean for each group using one-way analysis of variance (ANOVA) revealed a significant difference in mean number of risk factors dependent on diagnosis, $F(2,914)=57.05, \mathrm{p}=.001$ (refer to Table 14). The Asperger's Disorder group had a higher number of risk factors on average as compared to the other groups and the Autistic Disorder group had the least number of risk factors. The number of risk factors is summarized in Figure 5 based on a total of 917 responses and listed as the number of risk factors experienced. One participant did not answer any of the related questions and therefore was excluded from this analysis. 
Table 14

Number of Risk Factors per Group

\begin{tabular}{lccc} 
& & \multicolumn{2}{c}{ Group } \\
& Autistic & PDD-NOS & Asperger's \\
\cline { 2 - 4 }$M$ & 2.59 & 2.79 & 3.31 \\
SD & 1.928 & 1.912 & 2.018 \\
\hline
\end{tabular}


Figure 5

Total Number of Risk Factors

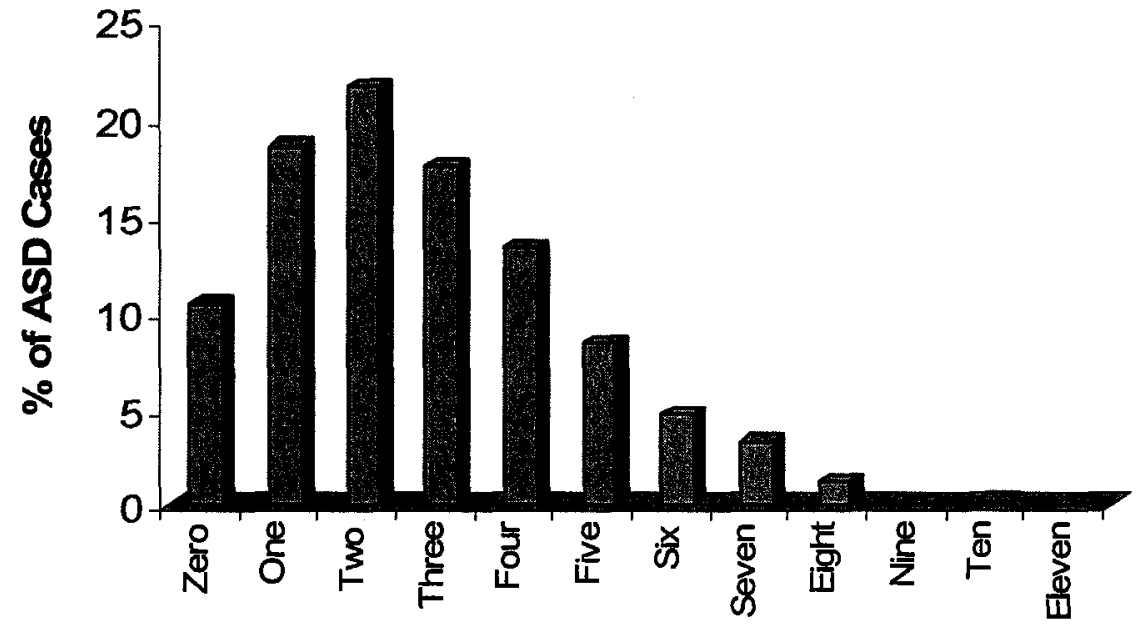

Risk Score 


\section{Severe Characteristics}

\section{Self-injurious Behaviours}

Participants were asked to identify if the individual with ASD displays any selfinjurious behaviours (SIB). A total of 906 respondents answered this question and 306 (33.8\%) indicated the occurrence of self-injurious behaviours. There was no significant difference between groups, $\chi 2(2, N=906)=4.39, p=.111$. The severity of SIB was coded as "mild", "moderate" or "severe". Inter-coder reliability was assessed on $25 \%(n=79)$ of the 289 cases with available data and was satisfactory at $86.5 \%$. The majority of cases in which SIB occur were mild in that they did not result in any visible marks on the body $(n=88,30.4 \%)$ or moderate and resulted in minor bruises or cuts and scratches $(n=128$, $44.3 \%)$. However, $25 \%(n=73)$ of cases were severe enough to cause scarring, require medical attention and result in the use of restrictive and intrusive interventions such as restraints or use of protective padding or a helmet. There was no significant difference between diagnostic groups in severity of SIB, $\chi 2(4, N=289)=4.67, p=.323$. However, it was of interest that 12 out of 73 severe cases of SIB had a diagnosis of Asperger's Disorder. The results are provided in Figure 6 and demonstrate that moderate SIB was most common in each diagnostic group. Gender was related to severity of self-injury, $\chi 2(2, N=289)=9.67, p<.05$, but not to presence of self-injury. In other words, girls are equally likely as boys, to show SIB but displayed the most severe SIB more often than was expected $(f=19, f e=13)$. 
Figure 6

Severity of Self-injurious Behaviour within Groups

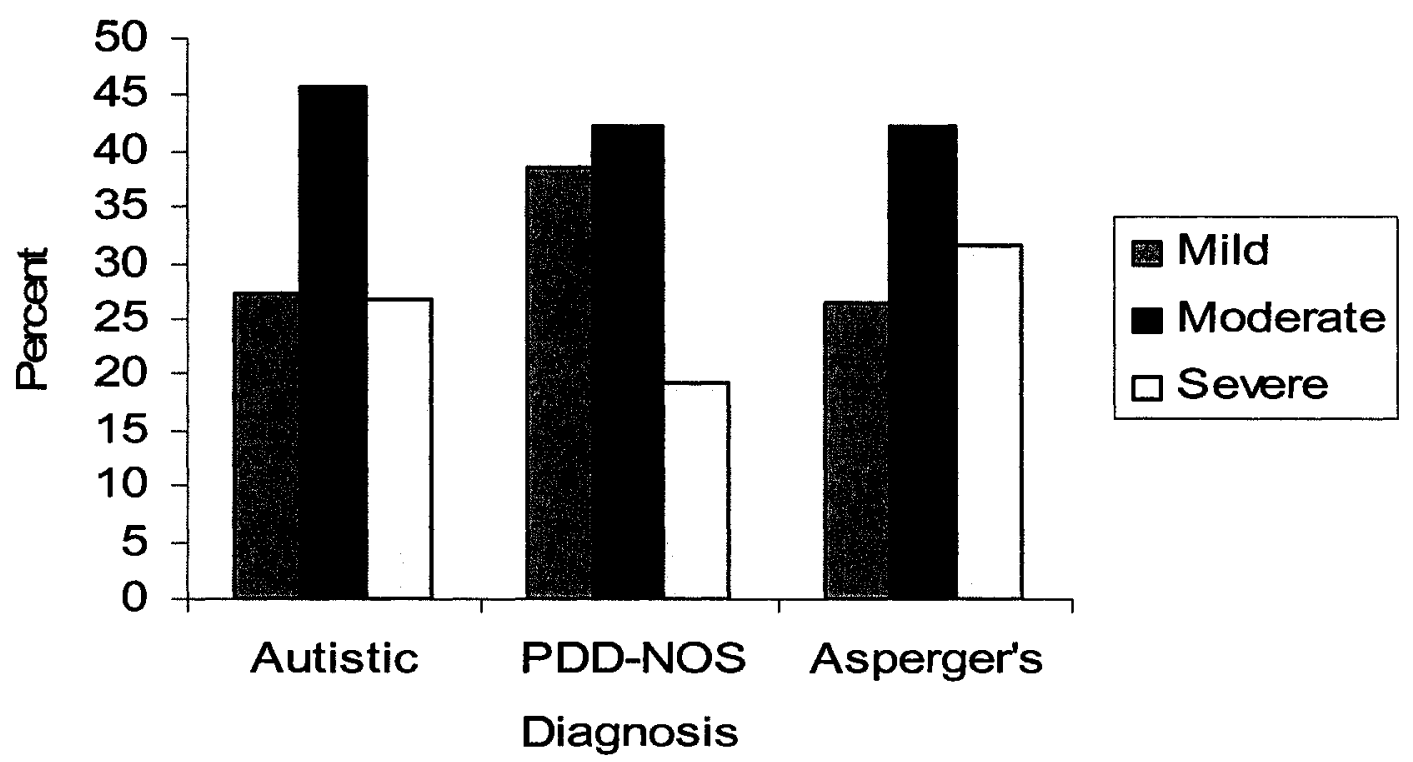




\section{Self-stimulatory Behaviours}

Participants were asked to indicate if their child with ASD displays any selfstimulatory behaviours. Out of 865 responses to this question, $80.3 \%(n=695)$ of the individuals with ASD do display some self-stimulatory behaviour. There was a significant relationship between diagnosis and presence of self-stimulatory behaviour, $\chi^{2}(2, N$ $=865)=12.20, p<.05$. In particular, the children with PDD-NOS $(f=212, f e=208)$ and Autistic Disorder $(f=390, f e=378.4)$ groups were more likely to display self-stimulatory behaviour than expected with $82 \%$ of each these groups displaying self-stimulatory behaviours. The individuals in the Asperger's Disorder group were less likely to have self-stimulatory behaviour than expected $(f=93, f e=107.7)$ with $69.4 \%$ of this group displaying the behaviours. No gender differences in the presence of self-stimulatory behaviour were found.

\section{Communication}

Survey respondents were asked to indicate what form of communication their child with ASD uses. These responses were categorized as exclusively verbal, sign language, use of picture systems or computer synthesized voice output units, a combination of verbal and augmentative communication, use of gestures or no functional system. The results are summarized in Table 15 and are reported as the frequency and percentage within each diagnostic group. The Asperger's Disorder group were almost exclusively verbal communicators, whereas, $42 \%$ of the Autistic Disorder group relied on augmentative communication or had no system of communication. The relationship between diagnosis and type of communication system was significant, $\chi 2(10, N=902)=71.87, p=.000$. No gender differences were found. 
Table 15

Primary Type of Communication System by Diagnosis

\begin{tabular}{lccc}
\hline & Autistic & PDD-NOS & Asperger's \\
& $n(\%)$ & $n(\%)$ & $n(\%)$ \\
Verbal & $282(58.1 \%)$ & $212(77.1 \%)$ & $128(90.1 \%)$ \\
Sign Language & $16(3.3 \%)$ & $5(1.8 \%)$ & $1(0.7 \%)$ \\
Picture System & $33(6.8 \%)$ & $8(2.9 \%)$ & $2(1.4 \%)$ \\
Combined & $92(19.0 \%)$ & $36(13.1 \%)$ & $6(4.2 \%)$ \\
Gestures & $44(9.1 \%)$ & $5(1.8 \%)$ & $4(2.8 \%)$ \\
No System & $18(3.7 \%)$ & $9(3.3 \%)$ & $1(0.7 \%)$ \\
Total within Group & $485(100 \%)$ & $275(100 \%)$ & $142(100 \%)$ \\
\hline
\end{tabular}




\section{Sleep Problems}

Participants indicated that half of the children with ASD display some form of sleep disorder or disturbance $(n=437 ; 50 \%)$. Furthermore, there is a significant relationship between diagnosis and sleep disorders, $\chi 2(2, N=874)=9.22, p \leq .01$, in that children with Asperger's $(f=77, f e=70.5)$ and Autistic Disorder $(f=248, f e=234)$ were both more likely to have sleep disorders than expected and individuals with PDD-NOS $f$ $=112, f e=132.5)$ were less likely than expected to have some form of sleep disturbance. Different types of sleep disorders were reported across the sample of ASD cases and the most common was awakening during the night, followed by irregular sleep times or patterns (see Figure 7). The type of sleep disorders were analyzed separately and were compared across groups. The results of these analyses are summarized in Table 16 and show that problems with awakenings and "other" sleep disorders vary depending on diagnosis while other types of disorders are equally common across all diagnoses. A significant relationship between gender and presence of sleep disorders was found, $\chi 2(1, N$ $=874)=5.84, p<.05$ with females reported as having a sleep disorder more often than expected $(f=89, f e=75.5)$. 
Figure 7

Percentage of ASD Cases with Specific Types of Sleep Disorders

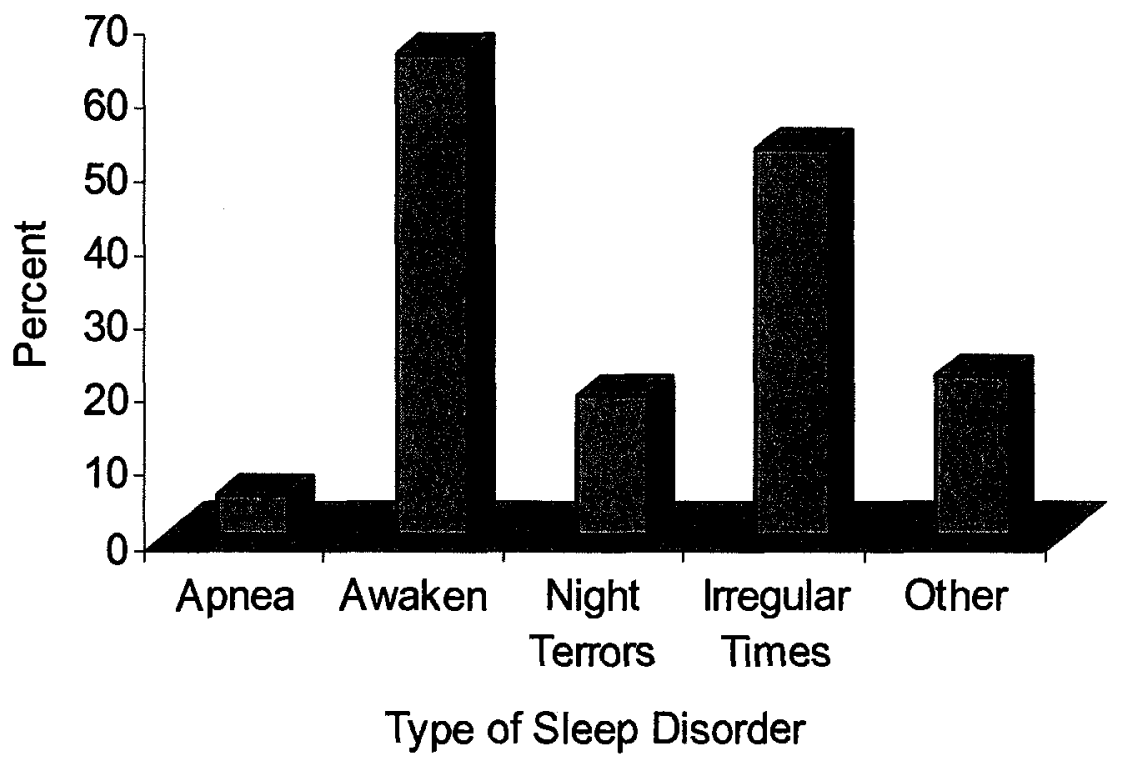


Table 16

Presence of Sleep Disorders in each Diagnostic Group

\begin{tabular}{lcccc}
\hline & $\begin{array}{c}\text { Autistic } \\
f(f e)\end{array}$ & $\begin{array}{c}\text { PDD-NOS } \\
f(f e)\end{array}$ & $\begin{array}{c}\text { Asperger's } \\
f(f e)\end{array}$ & $\chi^{2}(\mathrm{df})$ \\
\cline { 2 - 5 } $\begin{array}{l}\text { Presence of } \\
\text { Any Sleep }\end{array}$ & $248(234)$ & $112(132.5)$ & $77(70.5)$ & $9.217(2)^{*}$ \\
$\begin{array}{l}\text { Problem } \\
\text { Apnea }\end{array}$ & $16(14.3)$ & $8(6.3)$ & $1(4.4)$ & $3.464(2)$ \\
Awakenings & $179(166.2)$ & $71(73)$ & $40(50.8)$ & $9.621(2)^{*}$ \\
Night Terrors & $40(48.7)$ & $27(21.4)$ & $18(14.9)$ & $4.539(2)$ \\
Irregular Times & $143(134.7)$ & $49(59.1)$ & $43(41.2)$ & $4.950(2)$ \\
Other & $45(56.2)$ & $23(24.7)$ & $30(17.2)$ & $15.262(2)^{* *}$ \\
\hline $\begin{array}{l}* \\
* *\end{array} \leq .01$ & & & & \\
$* 001$ & & & &
\end{tabular}




\section{Risk Factors Related to Severe Characteristics}

Four risk factors including obstetric complications, comorbid medical conditions, family history of ASD and family history of mental illness, were examined for possible relationship to the four child characteristics of SIB, self-stimulatory behaviour, communication type and presence of sleep disorders. All analyses employed chi-square comparisons of categorical data. The results are described below and the relationships are summarized in Table 17.

Having experienced an obstetric complication was related to the presence of selfinjurious behaviour and sleep disorders. A significant relationship was found between the presence of obstetric complications and the presence of self-injurious behaviour, $\chi 2(1, N$ $=899)=13.07, p<.001$. A total of $39.2 \%$ of individuals with ASD who had experienced one or more obstetric complications also had SIB and this was more than expected $(f=183$, $f e=157.4)$. There was no relationship between obstetric complications and severity of SIB, $\chi 2(2, N=287)=0.84, p=.657$. A second significant relationship exists between obstetric complications and the presence of a sleep disorder, $\chi 2(1, N=867]=11.31, p \leq .001$. Individuals who experienced obstetric complications were more likely to also have sleep disorders $(55.6 \%)$ than would be expected if there was no relationship $(f=249, f e=$ 224.3). There were no significant relationships between obstetric complications and selfstimulatory behaviour, $\chi 2(1, N=858)=1.06, p=.302$, or type of communication, $\chi 2(5, N$ $=895)=1.40, p=.924$.

Having a comorbid medical condition was related to four different child characteristics including the presence of SIB, the severity of SIB, the presence of selfstimulatory behaviour and the presence of a sleep disorder. Individuals with ASD who 
Table 17

Summary of Relationships between Risk Factors and Child Characteristics

\begin{tabular}{|c|c|c|c|}
\hline Risk Factor & Child Characteristic & $\begin{array}{c}\text { Significant } \\
\text { Relationship }\end{array}$ & $\begin{array}{c}\text { No Significant } \\
\text { Relationship }\end{array}$ \\
\hline \multirow[t]{5}{*}{ Obstetric Complications } & SIB & $\mathrm{X}$ & \\
\hline & Severity of SIB & & $\mathrm{X}$ \\
\hline & Self-stimulation & & $\bar{X}$ \\
\hline & Communication Type & & $\mathrm{X}$ \\
\hline & Sleep Disorders & $\mathrm{X}$ & \\
\hline \multirow[t]{5}{*}{ Medical Condition } & SIB & $\mathrm{X}$ & \\
\hline & Severity of SIB & $\mathrm{X}$ & \\
\hline & Self-stimulation & $\mathrm{X}$ & \\
\hline & Communication Type & & $\mathrm{X}$ \\
\hline & Sleep Disorders & $\mathrm{X}$ & \\
\hline \multirow[t]{5}{*}{ Family History of ASD } & SIB & $\mathrm{X}$ & \\
\hline & Severity of SIB & & $\mathrm{X}$ \\
\hline & Self-stimulation & & $\mathrm{X}$ \\
\hline & Communication Type & & $\mathrm{X}$ \\
\hline & Sleep Disorders & & $\mathrm{X}$ \\
\hline \multirow{5}{*}{$\begin{array}{l}\text { Family History of } \\
\text { Mental Illness }\end{array}$} & SIB & $\mathrm{X}$ & \\
\hline & Severity of SIB & & $\mathrm{X}$ \\
\hline & \begin{tabular}{|l|} 
Self-stimulation \\
\end{tabular} & $\bar{X}$ & \\
\hline & Communication Type & & $\mathrm{X}$ \\
\hline & Sleep Disorders & $\bar{X}$ & \\
\hline
\end{tabular}


have a comorbid medical condition $(\mathrm{N}=364)$ were more likely to have SIB than expected $(f=140, f e=123.1)$. A total of $38.5 \%$ of individuals with comorbid medical conditions also had SIB and the relationship between the two variables was significant, $\chi 2(1, N=$ $816)=6.32, p<.05$. There was also a significant relationship between comorbid medical conditions and severity of SIB, $\chi 2(2, N=260)=10.30, p<.01$. Individuals who have a comorbid medical condition $(N=131)$ were more likely than expected to display the most severe forms of SIB $(f=45, f e=33.8)$ and these cases represented $34.4 \%$ of individuals who have comorbid conditions. Self-stimulatory behaviour was also related to having a comorbid medical condition, $\chi 2(1, N=785)=5.50, p<0.05$, in that $83.1 \%$ of individuals with ASD and a comorbid condition also displayed self-stimulatory behaviour. This represented a greater portion of the sample than expected $(f=285, f e=271.8)$. Sleep disorders were also related to comorbid conditions, $\chi 2(1, N=789)=31.83, p<.001$, with $61.6 \%$ of individuals with a comorbid condition also displaying a sleep disorder $f=221$, $f e=181.5)$. There was no relationship between comorbid medical conditions and type of communication, $\chi 2(5, N=813)=0.57, p=.989$.

A positive family history of ASD was related to the presence of SIB but not other child characteristics. Individuals with ASD who had at least one other relative with an $\operatorname{ASD}(N=241)$ were more likely to display SIB than was expected $(f=94, f e=81.2)$. This relationship was significant, $\chi 2(1, N=879)=4.22, p<.05$, but there was no relationship between family history of ASD and the severity of the SIB, $\chi 2(2, N=281)=$ $1.96, p=.376$. Likewise, there was no relationship between family history of ASD and self-stimulatory behaviour, $\chi 2(1, N=839)=1.86, p=.173$, type of communication, $\chi 2(5$, $N=875)=6.33, p=.276$, nor sleep disorders, $\chi 2(1, N=850)=1.49, p=.223$. 
A positive family history of mental illness was related to three child characteristics. First, family history of mental illness was related to SIB, $\chi 2(1, N=856)=14.17, p<.001$, but not the severity of SIB, $\chi 2(2, N=273)=0.53, p=.527$. In particular, individuals with ASD who had a family history of mental illness were more likely than expected to display $\operatorname{SIB}(f=168, f e=142)$. Of the individuals who had a history of mental illness, $84.1 \%$ displayed self-stimulatory behaviours and this was more frequent than expected $(f=344, f e$ =328.7). The relationship between family history of mental illness and self-stimulatory behaviour was significant, $\chi 2(1, N=815)=7.28, p<.01$, as was the relationship between family history of mental illness and presence of sleep disorders, $\chi 2(1, N=827)=10.02, p$ $=.01$. Those with a positive history $(n=416)$ were likely to have sleep disorders as well in $55.3 \%$ of the cases and this was higher than expected $(f=230, f e=207.2)$. Communication type was not related to family history of mental illness, $\chi 2(5, N=852)=$ $8.70, p=.121$.

\section{Discussion}

The identification of factors associated with Autism Spectrum Disorders is critical to understanding and predicting who is at risk for these disorders. The data used in this retrospective investigation supports the validity of the sample, and therefore the results, in several ways. First, the data is reported by parents. Parents know their children best and have the most comprehensive history of the experiences, family history and day-to-day behaviours of their children. Second, the participants were not directly approached or influenced to participate by service providers, therefore, eliminating referral bias and suggesting that parents freely devoted their time to respond to the questionnaire. The 
anonymity of an online survey may have been particularly helpful in making parents comfortable to report honestly. Parents were very forthcoming with very sensitive information about their children and families. For example, parents reported a wide range of mental illnesses in family members, described the medical details of their pregnancy and delivery and provided many personal details of their children's behaviours that presumably would be painful to recount. Third, the accuracy of the reporting of an ASD diagnosis is supported by the fact that participants were recruited through online sites and distribution lists of parent organizations for ASD. There would be no reasonable motivation for a parent to report a diagnosis of ASD and to frequent these sites if in fact their child did not have a diagnosis of ASD. The accuracy of the diagnoses was further supported by the reported average of two assessment tools used for making the diagnosis and by the fact that parents were often able to provide the names of these tools, the number of hours of observation leading to a diagnosis and the specific results of the various assessments. Based on prevalence estimates for the three diagnostic categories, PDD-NOS $(20.8 / 10,000)$ typically accounts for the largest portion of ASDs, followed by Autistic Disorder $(13 / 10,000)$ and Asperger's Disorder (4.3/10,000) (Fombonne, 2005b). The current sample had proportionally more cases of Autistic Disorder than PDD-NOS which may limit the inference of findings to the ASD population.

This data set was rich with detail and provided the opportunity to report on a wide range of factors, characteristics and relationships that make a valuable contribution to the existing body of literature. Several associated factors and severe characteristics were frequently experienced by individuals diagnosed with an ASD and the evidence strongly supports a genetic component to the development of the disorder. Some significant 
relationships between various factors and specific diagnoses were also found. Ultimately, these differences in risk factors may one day help to identify children at risk at an earlier age than currently possible and result in earlier intervention, thus leading to better outcomes for individuals and families.

Participants

Internet data collection facilitated access to a large sample of families affected by autism. Previous studies have typically been based on small samples and often, geographically restricted areas. This sample included 918 participants and each diagnostic group contained enough participants to lend adequate statistical power to the analyses. The sample was primarily North American and a broad perspective on the prevalence of these experiences and characteristics was gained. There were no significant relationships between place of residence and any of the factors under investigation which supports the validity of the sample. The total number of responses from countries outside of the U.S.A. and Canada were small and therefore, the accuracy of any inference to the broader ASD population is limited.

The gender ratio of 4.9 boys for every 1 girl in this sample is comparable to the typical ratio reported in epidemiological studies (Fombonne, 2003) and in this study there was no significant relationship was found between gender and diagnostic groups. Consistent with previous research, these findings support that boys are at greater risk for ASD than are girls. However, the results of this study suggest that amongst those who have ASD, it is girls who may be more likely to experience some of the most serious symptoms including comorbid medical conditions, sleep disorders and severe self- 
injurious behaviours. These gender differences may be due to sex-linked heritability factors or perhaps differences in genetic susceptibility to environmental influences.

The online format of this parent-report questionnaire for collecting this data presented a limitation in terms of confirming the accuracy of a diagnosis. However, this was primarily a young sample of participants at the time of the survey completion which improves confidence in the comparability of the group because diagnoses were likely to be based on a recent set of criteria in the DSM-IV (APA, 1994) or ICD-10 (WHO, 1993). Although the DSM-IV is most common in North America, and the ICD-10 in Europe, the fact that place of residence was not related to any of the variables in this study, the impact of the differences between these diagnostic criteria is likely minimal in this study. In addition, it was likely that recent assessment instruments would have been used for the majority of the sample. Ideally, future studies would control for variation in diagnosis by confirming the diagnosis based on common assessment tools and independent verification.

The age at which the individuals were first diagnosed varied depending on diagnosis. Individuals in the Autistic Disorder group tended to be diagnosed earlier and with fewer hours of observation whereas, individuals in the Asperger's group tended to be diagnosed at an older age and had a higher number of observation hours during the assessment period. The symptoms are typically more apparent in a more severely affected child and so it seems that the children with Autistic Disorder would be likely to be diagnosed early and with less rigorous observation because of severe impairments in communication and behaviour. In comparison, the symptoms of Asperger's Disorder are generally more subtle due to relative strengths in language and intellectual functioning and may, therefore, not be obvious until the child is older and demands for social interaction 
and independence increase during the school years for example. An increased amount of observation may be warranted for this group to make a differential diagnosis based on these comparatively subtle characteristics.

It was common for individuals to experience a change in diagnosis (20\%) to either more or less severe on the spectrum or even to some other diagnosis. There are several possible explanations for this finding. First, it is possible that clinicians are reluctant to give families a specific diagnosis initially because of the devastating prognostic implications. A doctor may prefer to give a more general label of ASD or pervasive developmental disorder or simply, developmental delay, in attempt to soften the message for families. As the child gets a little older and families become more accepting of their child's problems, clinicians may provide a more specific diagnosis such as Autistic Disorder. Secondly, developmental progression may impact on the accuracy of diagnosis. A child who is relatively high functioning and is diagnosed at young age may show more of the autistic characteristics as they age and are exposed to environments such as preschools and schools where the social demands make it more obvious that they are not able to function at the same level as compared to their peers. Alternatively, if a child is diagnosed early and receives effective intervention, it is possible that they would no longer meet diagnostic criteria. Finally, it is plausible that misdiagnoses occur and are likely due to the difficulty in making a differential diagnosis given the complexity of the severe characteristics and comorbid conditions that have been reported. This last point suggests the need for ongoing research and development of precise tools and methods for making differential diagnoses. 
Data for this study was collected through an online questionnaire through autism distribution lists and parent organizations. This method was advantageous in reaching a wide variety of participants in an efficient manner and we can assume this was most likely reaching an autistic population rather than other diagnoses because the respondents were a part of these networks. However, there were some disadvantages. Participants would have to have had access to a computer, have adequate computer skills to complete the survey and communicate adequately in English in order to provide accurate responses to the questions, which is likely the reason behind the relatively high social class of this sample as reflected in the overall high family income. As a result, the representativeness of this sample to the broader population may be limited given that ASD occurs across all socio-economic classes (Fombonne 2005a; Tidmarsh \& Volkmar, 2003). In this study, the family income did not differ based on diagnostic category, therefore, does not limit the representativeness of this sample. Based on the experience of using this method of data collection, online surveys are useful for collecting large samples of clinical populations provided that adequate measures are taken to ensure the representation of the sample.

It is interesting to note that nearly $40 \%$ of mothers in this sample stay at home to care for the child(ren). The U.S. Department of Labor reported that in the year 2000, the rate of stay-at-home mothers was around 25\% (Bureau of Labor Statistics, 2000). Likewise, the Canadian trend for working mothers has increased over the years such that in $2005,23-33 \%$ of mothers, depending on the age of their children, were at home with their children full-time (Statistics Canada, 2006). The higher than expected number of stay-at-home mothers in this study, is likely the result of the increased demands of caring for a child with exceptional needs. Also, in the clinical experience of the author, parents 
often report that it is difficult to find appropriate childcare if they do wish to work outside the home.

Speculation about a link between autism and parents who work in the engineering field has resulted in several popular media reports about parents of children with ASD who work in the high technology industry. A scientific study found that $28 \%$ of fathers and grandfathers of children with ASD were working in the engineering field as compared to $15 \%$ of the control group (Baron-Cohen, Wheelwright, Stott, Bolton \& Goodyer, 1997). This is seen as support for the genetic link to ASD and the broader phenotype of the disorder, which, to the extent that these characteristics are relevant to career success in physics, can be an adaptive advantage. The results of the current study indicated that $15.3 \%$ of the fathers worked in the field of natural and applied sciences but this was not substantially higher than the rate of fathers that worked in social sciences $(14.7 \%)$ or in Trades $(12.5 \%)$. The results of the current study suggest that there is no definitive link between employment in natural and applied sciences. However, when treating and researching ASD, it is critical that individual strengths not be overlooked in favour of focussing on the skill deficits and impairments.

\section{Risk Factors}

The large sample in this study presented a valuable opportunity to investigate the frequency of various experiences of individuals who later were diagnosed with an ASD. Previous literature has typically focussed on a small number of such factors and usually with relatively small samples. These studies have identified a number of risk factors believed to be related to the disorder but very little is known about how common it is for individuals to have experienced these. This study is one of the first to map out a wide 
range of risk factors in a large sample and compare these experiences across specific diagnoses.

Several risk factors, believed to be indicators of genetic involvement in the disorder, were found to be commonly experienced by individuals with ASD in this study. These risk factors include comorbid medical conditions, having other relatives with an ASD, having other relatives with mental illnesses, and having older parents.

Comorbid medical conditions were common in this sample. Previous studies have estimated that medical syndromes or chromosomal abnormalities are present in $8-10 \%$ of ASD cases (Gillberg \& Cederlund, 2005; Rutter, 2005a; 2005b; Tharp, 2003) and Fombonne (2005b) reported a median rate of $5.5 \%$ in a review of studies reporting medical conditions associated with autism. In the present study, nearly $40 \%$ of ASD cases had a broadly defined, comorbid medical condition. The results reported in Table 3 show that "other" more mild and benign conditions were most common and it is important to consider that parents may have over-reported symptoms they consider to be of a medical nature because they were concerned about such things as ear infections, colds, flu symptoms or similar maladies, and not all of these reported symptoms would necessarily meet diagnostic criteria for a medical condition. Therefore, the representativeness of this data is suspect. The parent report about seizure activity or chromosomal conditions is likely to have been more representative as parents would be unlikely to over-report these serious conditions that require medical confirmation of diagnosis. The specific chromosomal conditions were present in $4.3 \%$ of cases and neurological conditions were present in $20.9 \%$ of cases. The high frequency with which medical conditions, including major organ disease or dysfunction, occurred in this sample is suggestive of systemically 
compromised overall health for at least a subset of individuals with ASD. Whether this is due to genetic inheritance or spontaneous chromosomal mutation or other factors, is worthy of further scientific investigation.

It is common to hear discussion about gastro-intestinal symptoms being associated with ASD and although only $10 \%$ of this sample reported these symptoms, it does warrant further study as previous reports indicate that families are commonly using non-evidence based treatments such as restrictive diets, nutritional supplements and medications to treat these symptoms (Green et al., 2006; Hicks, Bowker \& Wells, 2006). Similarly, mental health conditions were present in a sufficient number of ASD cases $(9.8 \%)$ and particularly in Asperger's cases (22.2\%) to warrant the careful attention of practitioners treating individuals. This study highlights the complexity of medical issues that exist outside of the core diagnostic criteria for ASD for many individuals. Given the prevalence of comorbid conditions, it is imperative that practitioners thoroughly investigate medical and mental health conditions on an individual basis with clients identified with an ASD and provide appropriate treatment for any existing comorbid conditions. A great deal of scientific investigation as to the cause of these conditions is still warranted and these studies will lead to better understanding of the possible genetic influences on the development of ASD.

Family history of ASD and mental illness seem to be important risk factors for ASD. Other studies have suggested the broader phenotype of ASD is present in 10-20\% of first degree relatives (Rutter, 2005b) and siblings are particularly at increased risk for the disorder (Bolton et al., 1994). The possible inheritance of ASD is further supported by the current study's finding that more than a quarter of this sample had at least one 
additional case of ASD in the immediate or extended family although it was relatively rare in the sample that families included more than 2 cases of ASD. Furthermore, half (49.3\%) of this ASD sample had relatives with mental illnesses such as schizophrenia, depression, anxiety, bipolar disorder and obsessive compulsive disorder. The rate of mental illness in the general population is reported as $26 \%$ of adults in the U.S. (Kessler, Chiu, Demler \& Walters, 2005) and 20\% in Canada (Health Canada, 2002) indicating that the prevalence of mental illness of families affected by ASD is double the norm according to parent reported data in this study. Disorders of mental health have long been of interest and have often been studied as possible correlates to or risk-factors for ASD (Bolton, Pickles, Murphy \& Rutter, 1998; Mikali, Chakrabarti \& Fombonne, 2004; Mouridsen, Rich, Isager \& Nedergaard, 2007) although samples have been small and narrow in focus, in comparison to the present study. Without a control group for comparison of the current findings, inference to the ASD population is limited but does highlight the need for further investigation. Researchers should utilize control groups in future studies to compare the prevalence of such disorders in children with ASD with children who are developing normally and children who have other types of disabilities. Also, since $73 \%$ did not have other relatives with ASD and 50.7\% did not have relatives with identified mental illnesses, it is unlikely that genetic factors are the only risk factors for ASD and other factors such as environmental insult or injury are possibly important.

Researchers generally agree that advanced parental age can put a child at increased risk for disorders including ASD but studies so far have found conflicting results as to whether maternal age or paternal age or both are important. The present sample had a higher mean age of mothers as compared to the American national average of 27.2 years 
in 2000 (Matthews \& Hamilton, 2002); and a higher mean age of both parents compared to a previous study by Williams, Oliver, Allard and Sears (2003). The mean age of mothers in this study was 30.4 years compared to 26.3 years from Williams et al.. The mean age of fathers in this study was 32.6 years compared to 28.9 years from the Williams et al. study. Furthermore, $23.7 \%$ of mothers were older than 35 years at the time of the birth of the child with ASD, as were $34.4 \%$ of fathers in this study. Advanced maternal age may increase risk for pregnancy or birth complications and therefore injury or insult to the child (Glasson et al., 2004). However, advanced paternal age may indicate the child is at increased risk for chromosomal mutation leading to ASD (Reichenberg et al., 2006). The present study provides evidence that advanced maternal and paternal age is common in a large sample of ASD cases and should receive further attention from scientists. Over the past 30 years, the trend is towards couples delaying the start of their families and commonly having children at an older average age than ever before (Matthews \& Hamilton, 2002). Given the increased prevalence of ASD in recent years, this correlation should be carefully investigated and if the evidence supports this as a risk factor, awareness campaigns should be promoted to educate parents-to-be on the risks and the early signs of ASD.

Obstetric complications were present in just over half of all cases of ASD in this study. In a study comparing autistic children with a matched control group, researchers found complications in $35 \%$ of the control group and the difference between controls and the autistic group was not significant (Cryan, Byrne, O'Donovan \& O'Callaghan, 1996). By comparison, the current finding that $50 \%$ of ASD cases have experienced complications is higher. It is unclear whether these complications are due to pre-existing 
genetic differences which also relate to the development of ASD or if these are accidents and insults that occur for other reasons and subsequently trigger susceptibility for the disorder, or ultimately cause the symptoms of ASD. Complications of labour and delivery were most common and may result in injury or deprivation of oxygen leading to brain damage that in theory may be responsible for the onset of ASD. The same is possible in the case of maternal illness, injury or use of medications which was present in $28 \%$ of cases. Neonatal health problems occurred in $14 \%$ of the cases reporting complications. It is not yet clear whether this is due to systemically compromised health including ASD or whether these early health complications trigger the disorder. Less than optimal gestational age at delivery occurred in $27.8 \%$ of the ASD cases when preterm and overdue cases were combined. By comparison, the Canadian rate of premature birth was $6.8 \%$ in 1994 (Joseph et al., 1999) which was around the time that most of the children in the current study were born. The U.S. National rate of premature births was just over $12 \%$ in 2005 (National Center for Health Statistics, 2007) so the finding that children were born premature in $17.5 \%$ of the present ASD sample appears to be much higher than the norm. Whether this is a correlate or a potential cause of ASD remains unclear but certainly warrants consideration as a risk factor given that a recent study of 91 preterm infants found that $26 \%$ of these infants scored positively on a screening measure for autism (Limperopoulos, et al. (2008). Parents who experience complications during pregnancy, including preterm labour or delivery should be educated about watching for possible early signs of ASD in their child and medical practitioners should carefully screen the child for developmental milestones and "red flags" of ASD. 
It was hypothesized that risk factors may have some cumulative effect in triggering or causing ASD. The available data was categorical and therefore, a risk score was derived by combining points for advanced parental age, family history of mental illness, family history of ASD and comorbid medical conditions which were all found to be common in this sample. The analyses were simplistic but indicated that on average, people with ASD have had 2.76 of these experiences. Therefore, children who experience more than one of these individual factors may possibly be at increased risk for the disorder and thus, these factors could be assessed and monitored by paediatricians to help identify children at an early age and stage of development. Although it is important not to alarm parents unnecessarily, it would be helpful to provide general education about watching for signs of healthy development in young children which include communication, social development and behaviours. Further study is necessary to identify whether the number of risk factors or the relative importance of type of risk factor is more important.

\section{Risks Related to Diagnoses}

A comparison of various risk factors across specific categories of diagnosis was undertaken and while some did not differ, there were a number of risk factors that did vary depending on specific diagnoses. The risk factors that were not related to specific diagnosis include presence of medical conditions in general, gastro-intestinal symptoms, parental age, or presence and type of obstetric complication. A relationship was significant between diagnoses and type of medical condition, family history of ASD, family history of mental illness, birth order, gestational age at birth and total number of risk factors. 
Some of the most interesting relationships between diagnoses and risk factors involved Asperger's Disorder specifically. The present results seem to support that having a chromosomal or neurological condition is an associated risk factor for the more severe end of the ASD spectrum as compared to Asperger's Disorder. However, individuals who have Asperger's frequently have accompanying mental health problems which are perhaps not an indication of risk for the disorder, but more likely a result of the social isolation and frustration associated with the social impairments that are characteristic of the diagnosis. On the other hand, the results also indicated that family history of mental illness is especially likely in the Asperger's group and so, mental health problems may be a sign of genetic risk. It was also most common that individuals with Asperger's had at least one other relative with ASD. This could mean that Asperger's has a greater likelihood of genetic risk or, possibly, that once a case of ASD is identified in a family, relatives are more aware of the disorder and there is an increased likelihood that the more subtle features of Asperger's Disorder are recognized and this leads to more diagnoses. Regardless, based on these findings, parents and couples thinking of becoming parents, who have a strong family history of mental illness and / or ASD should be made aware of this potential risk and be counselled to watch for early indications that may warrant an assessment.

Although birth order followed a similar pattern across diagnostic groups, the Asperger's group was especially likely to be first born and the Autistic group had an increased rate of being born later. The meaning and potential importance of this finding is unclear but may indicate that first pregnancies are at increased risk for Asperger's and that risk for Autistic Disorder increases with subsequent births. Also, children with Asperger's 
were more commonly born post-mature and children with Autistic Disorder were more often premature. One might assume that being born too early increases the potential for more severe damage that is associated with Autistic Disorder. Alternatively, the more profoundly affected foetus with Autistic Disorder and possibly other medical symptoms may be more likely to be born premature. Lastly, the total number of risk factors differed based on diagnosis and surprisingly, it was the Asperger's group who had experienced the highest average number of risk factors. Given that Asperger's disorder is at the least severe end of the autism spectrum, the type of risk factor is likely more relevant than the total number of risk factors. It is also possible that the most relevant risk factors were not captured in this analysis and some other combination or weighting of variables would produce a more meaningful finding.

\section{Severe Characteristics}

There is limited research available that reports how common it is for individuals with ASD to present with some of the most concerning and severe symptoms associated with the disorder. This study investigated four such symptoms, including self-injurious behaviour (SIB), self-stimulatory behaviour, mode of communication and sleep disorders. Intellectual functioning was of interest as well but was not analyzed in this study due to the unreliable nature of the parent reported description of results.

One of the most interesting results in this study was that SIB was present in $33.8 \%$ of the sample and did not differ significantly by diagnosis meaning that even the individuals with Asperger's presented with SIB. Another study has reported a similar rate of SIB in a smaller sample (Dominick et al., 2007) and the rate was higher at $53 \%$ in one autistic sample (Baghdadli, Pascal, Grisi \& Aussilloux, 2003). Although most of the SIB 
was mild or moderate in the current sample, $25 \%$ of the cases reporting SIB were severe enough to cause injury, require medical attention or intrusive measures were necessary to protect the individuals from harming themselves. It also appears that girls with ASD may be at increased risk for the more severe forms of self-injury.

Self-stimulatory behaviours can interfere with learning, draw negative attention that may restrict social opportunities and repetitive motion may cause strain or tissue damage over time. These behaviours were reported by parents to be present in $80 \%$ of the overall group of ASD cases. Not surprisingly, the Asperger's group had a lower rate of self-stimulation than did the Autistic and PDD-NOS groups. It would be interesting for future studies to investigate differences in type of stimulatory behaviour as it is likely that individuals with Asperger's are more likely to engage in verbal repetition of topics and questions as opposed to the more physical symptoms of rocking, hand-flapping or twirling that may be more common for individuals at the more severe end of the spectrum.

The means by which a person communicates is not an indication of intelligence but in the ASD population, augmentative communication is often used. When an individual relies on an augmentative communication system or has no formal system to communicate, this can result in marginalization and increased complications in independent functioning. As anticipated, the exclusive use of verbal communication was more frequent at the less severe end of the spectrum and augmentative communication was more frequent at the more severe end. A few individuals with Asperger's Disorder reported the use of augmentative communication tools and it is unclear if this is due to impairments related to the disorder or if there may be additional reasons such as motor or hearing impairments that restrict verbal communication. The other possibility is an 
inaccurate diagnosis of Asperger's Disorder. A range of augmentative systems were used including sign language, picture systems, gestures or a combination of verbal and augmentative communication. Although the number of individuals with no system to communicate was small $(n=28)$, this is still very concerning as previous literature has indicated these individuals are at greater risk for SIB and aggression. The limited data available in this data, limited the analyses to categorizing the primary mode of communication. Future studies could investigate the range of functional communication. For example, a person may use sign language as their primary mode of communication but only use 5 signs to request their most preferred toys or foods and is therefore very limited in their functional communication.

Sleep problems can have a huge impact on the individual as well as the family. In this sample, sleep disorders were reported by parents for $50 \%$ of the individuals with ASD and the rate differed significantly based on specific diagnoses. This rate is double the usual report of sleep disorders in childhood in that Western countries have typically reported rates of between $20-25 \%$ for children's sleep disorders (Mindell, Owens, \& Carskadon, 1999). Similarly, in a recent Chinese study using parent survey for data collection from the general population, the prevalence rate of sleep disorders in 2 to 12 year olds was $21 \%$ (Liu et al., 2005). Researchers who studied sleep disorders in an autistic sample have reported rates of sleep problems as high as $83 \%$ with problems related to falling asleep and staying asleep commonly reported (Wiggs \& Stores, 2004). Similarly, in the present study, irregular sleep times, patterns and awakenings were the most commonly reported sleep problems. Individuals in the Asperger's group were particularly likely to experience "other" sleep problems such as difficulty falling asleep and were less likely to have 
frequent awakenings. The Autistic group reported trouble with night awakenings more often than expected. Girls with ASD were more likely to have sleep disorders and this relationship between gender and sleep disorders mirrors findings in the general population of children (Liu et al. 2005). For all groups, sleep problems can result in low performance and energy throughout the day due to fatigue and use of effective treatments needs to be considered. In particular, previous research has found sleep disorders are associated with increased risk for behaviour problems (Minde et al., 1993). Given that individuals with autism commonly experience sleep problems, clinicians should be prepared to assess and treat these problems.

\section{Risk Factors Related to Severe Characteristics}

A variety of severe symptoms are often experienced by individuals with ASD and research about risk factors for these symptoms has been very limited. Self-injury is a very concerning behaviour and is perhaps one of the most severe symptoms that occur in this population. Behavioural treatment approaches to SIB have been successful in many cases (Matson \& LoVullo, 2008), but ideally, these behaviours would be prevented rather than treated. This requires early identification of those at risk for extreme behavioural characteristics and this study has identified four risk factors related to severe characteristics. Children with ASD who have a comorbid medical condition, a family history of mental illness, a family history of ASD, or experienced obstetric complications appear to be at increased risk for self-injurious behaviour. Sleep disorders are associated with family history of mental illness, comorbid medical conditions and obstetric complications. Self-stimulation is related to comorbid medical conditions, and family history of mental illness. 
The associations between risks for ASD and severe characteristics are indicative of genetic risk for the disorder and have important implications for clinical practice. Individuals with ASD show one or more severe symptoms in $34-83 \%$ of cases if they also have a medical condition; $40-55 \%$ of cases when an obstetric complication was experienced; $39 \%$ of cases that have a family history of ASD; and, $40-84 \%$ of cases with a family history of mental illness. These relationships indicate family and medical history are very important to the development of severe behavioural characteristics in at least 1 in 3 ASD cases. The frequencies of compromised health and positive family history of related problems in this sample implicate the role that genetics play in the development and manifestation of ASD. The frequency with which these associated factors occur has serious implications for clinical practice and careful consideration in treatment planning must be undertaken.

Clinicians should consider family and medical history carefully in developing and monitoring patient care and proactively identify children at increased risk for such extreme behavioural manifestations. Often children develop behaviours such as self-injury or selfstimulation, due to their lack functional communication, play skills and social interaction abilities. Early access to high quality treatment programs to develop these skills and effectively manage challenging behaviours if they occur is necessary to minimize these behaviours. The use of Applied Behavioural Analysis has been effective in enhancing the skills of children with ASD and is currently indicated as the most effective treatment (New York State Department of Health Early Intervention Program, 1999). Future research should investigate the relative influence of various risk factors on severe characteristics. 


\section{Implications}

This study was an important contribution to the body of literature on ASD and risk factors in several ways. By identifying how common it is for individuals with ASD to have experienced perceived risk factors, researchers can narrow their focus on particular factors and groups to try to find the cause of the disorder. These potential risk factors are largely unpreventable, but the knowledge that they present a risk is useful in educating parents and practitioners and may lead to earlier and more accurate diagnosis in the future which has the potential to lead to better outcomes for children. Practitioners can use these findings in their screening, identification and treatment of individuals with ASD by assessing the risks that a given child has experienced and following these children more closely to watch for early signs of ASD. Parents too can be counseled as to potential risks and educated about the importance of seeking developmental screening and assessments if warranted. For example, parents-to-be who are over the age of 35 , have family histories of mental illness and / or ASD could be informed that these associated factors may increase their risk for having a child with ASD and be provided with training in recognizing when to seek professional consultation about developmental concerns. Parents who seek advice prior to conception may also make more informed decisions about having children. In recent years, couples are having children later in life than ever before and reproductive technologies are making this an increasingly viable option and it is worth considering that this may put more families at risk of ASD. The potential risks for ASD are a long way from being fully understood, therefore, should be interpreted cautiously and sensitively shared with the public and parents-to-be so as to avoid unwarranted fears and anxieties. 
Some important lessons were learned through the use of an online questionnaire format for data collection in this study. The major advantage of using the online format was the ability to reach a large international sample with relative ease, efficiency and low cost. The size of the sample was critical to the value of this study in that it is rare to have a large sample size in this field. The large sample created a rare opportunity for comparison across specific diagnostic categories. However, the online data collection by parent report was not optimal in a number of ways and while specific limitations are discussed below, some of the general ethical issues of these research techniques are discussed here.

The reported increase in prevalence of ASD has prompted an increase in research and public awareness of the disorder. Families are, understandably, desperately searching for answers and for help for their children and are therefore vulnerable. Previous research has shown that many families either do not know or do not understand research or perhaps disregard it a desperate attempt to help their children. This is demonstrated by the common use of non-evidence-based treatments utilized by families for children with ASD (Green at al., 2006; Hicks, Bowker \& Wells, 2006).

The use of technology to reach research participants is valuable and ever increasing but poses ethical concerns, particularly when involving vulnerable populations such as the ASD community. Brownlow and O'Dell (2002) have identified the need for professional guidelines for using internet technology in order to ensure that informed consent is gained and participant privacy is protected. Research related to the etiology of Autism is critical and due to the highly heritable nature of the disorder needs to include participants with ASD and their families (Chen, Miller \& Rosenstein, 2003). However, 
this type of research, including the current study, poses an ethical dilemma in that it relies on the participation of a vulnerable population with little or no direct benefit to them (2003). Therefore, a cost benefit analysis must be carefully considered prior to seeking participants from the ASD population. In the current study, participants' identities were protected in that no names were submitted and identifying information was limited. The questionnaire required limited time commitments and was not suggestive of direct benefits for participants, therefore, it seems the benefits out weigh the costs in this study and it was ethical to proceed.

If this study was repeated or a similar methodology was employed again, it would be useful to limit the descriptive data that was collected and to provide predetermined categories of responses for as many questions as possible. This would improve the accuracy of the reporting and the analyses and reduce participant time and effort.

\section{Limitations}

The results of the current study are limited by several factors. First, the study was conducted to investigate the prevalence of various factors in the ASD population that have been previously reported as possible risk factors and to explore differences between the specific diagnoses and also to investigate the prevalence of severe characteristics and relationships with specific diagnoses and gender. The use of the term "risk factors" may be misleading in the current study in that this was not a general population study investigating prospectively how many people who have these factors actually develop ASD. This was a retrospective look at how commonly various factors were experienced in a clinical sample and reports on factors associated with ASD. Second, the data were almost exclusively categorical and therefore, limited the available options for analyses. 
However, chi-square analyses were useful in revealing relationships between a number of factors and allow for some inference to the ASD population. The third limitation in this study is a result of the nature of a parent-report questionnaire. There was no confirmation of the accuracy of the diagnosis of participants and other reported information. Although there is no reason to suspect that respondents deliberately provided mis-information, parents may have been mistaken in their reporting, for example, about extended family histories or they may have misinterpreted various questions which may have lead to inaccurate reporting. As an online questionnaire posted only in English, the study unfortunately excluded participants who did not have access to computer technology or did not read English. Despite these limitations, the results are valuable in that they highlight various areas for ongoing research.

\section{Future Studies}

Ongoing research is critical to identify risk factors for ASD and to understand the possible relationships among various factors and the specific diagnoses and severe symptoms that are common. Prospective studies are needed to determine how likely it is for a child that has an individual risk factor or combination of risk factors to develop an ASD. Comparison of risk factors between groups is also needed such that ASD groups are compared to a control group of typically developing individuals and also to other disability groups as previous research has been contradictory in whether the risks are different for ASD than other disabilities (Klug et al., 2003; Matsuishi, 1999; Mouridsen, Rich, Isager \& Nedergaard, 2007; Williams, Oliver, Allard \& Sears, 2003). Methodologies would ideally include independent confirmation of diagnosis using the best available standardized measures for consistency, reliability and validity. Although survey data is useful, more 
rigorous designs will promote greater confidence in the results and limit bias in reporting. Much more research is required before the relationship between risks and ASD is fully understood.

\section{Concluding Remarks}

The cause of autism is still unknown. There are currently more children diagnosed with ASD than ever before and parents are desperate for answers and for help. Previous research has revealed a number of factors associated with the disorder which support the inheritance of the disorder or at least, a genetic susceptibility. These studies have often been limited in scope and used small samples. Very few have ever compared the experiences of children with Autistic Disorder, PDD-NOS and Asperger's Disorder. The current study was initiated to add to the current knowledge base on risk factors, severe characteristics and to explore differences between groups.

One goal of the current study was to report on how common various factors were experienced and to examine relationships between those experiences and the specific diagnoses on the autism spectrum. The results of this examination revealed that factors suggestive of genetic involvement in the disorder are commonly experienced in the ASD population. Furthermore, the results suggested that children at the more severe end of the spectrum experience different risk factors than those who have a diagnosis of Asperger's Disorder.

A second goal of the current study was to report on the frequency of severe characteristics associated with autism in general and across specific diagnostic categories and to examine relationships between risk factors for ASD and these severe characteristics. Self-injurious behaviour, self-stimulatory behaviour, sleep disorders and 
non-verbal communication were commonly reported in this sample of individuals with ASD. The results of these analyses highlight the complexity of issues to be addressed in the treatment of these individuals. It appears that individuals who have an ASD and familial history of mental illness or ASD are at increased risk for these severe characteristics, as are individuals who have experienced medical complications. These findings further support the genetic influences for the disorder in that there are associated familial factors and the comorbid medical complications can be seen as potential triggers of genetic susceptibility or signs of genetically comprised overall health.

There is a great deal of research still necessary to isolate the specific risks for autism. Results of this study generally supported previous research in this area. However, several questions remain that warrant further investigation. For instance, are the risk factors for the disorder reliably different for each category of the diagnosis on the spectrum? Also, are there different associated factors that have not been reported in the literature? Combinations of factors that might represent a critical 'genetic loading' that results in the expression of the disorder and that relates to the severity should also be considered. Alternatively, when genetic risks, such as family history of ASD, are combined with environmental insults, such as birth complications, does this increase risk for the disorder? The questions and possibilities are numerous. In the future, prospective studies with control groups for comparison and independent verification of participants' diagnoses will hopefully provide more answers. 


\section{References}

Akçakin, M., Polat, S. \& Kerimoglu, E. (1993). A comparison of demographic and birthrelated characteristics of autistic and intellectually handicapped children. Türk Psikiyatri Dergisi, 4, 39-46.

American Psychiatric Association (1994). Diagnostic and Statistical Manual of Mental Disorders IV. Washington, DC, Author.

American Psychiatric Association (2000). Diagnostic and Statistical Manual of Mental Disorders IV-TR (text revision). Washington, DC, Author.

Baghdadli, A., Pascal, C., Grisi, S. \& Aussilloux, C. (2003). Risk factors for selfinjurious behaviours among 222 young children with autistic disorders. Journal of Intellectual Disability Research, 47, 622-727.

Baron-Cohen, S. (1995). Mindblindness: An essay on autism and theory of mind. Boston: MIT Press/Bradford Books.

Baron-Cohen, S. (2001). Theory of mind and autism: A review. In L.M. Glidden (Ed), International review of research in mental retardation: Autism (pp. 169-184). San Diego: Academic Press.

Baron-Cohen, S., Leslie A.M., Frith, U. (1985). Does the autistic child have a 'theory of mind'? Cognition 21 (1): 37-46.

Baron-Cohen, S., Wheelwright, S., Lawson, J., Griffin, R. Ashwin, C. Billington, J. et al (2005). Empathizing and systematizing in autism spectrum conditions. In Volkmar, F.R., Paul, R., Klin, A. \& Cohen, E. (Eds) Handbook of autism and pervasive developmental disorders (pp. 628-639). New Jersey, John Wiley \& Sons, Inc. 
Baron-Cohen, S., Wheelwright, S., Stott, C., Bolton, P. \& Goodyer, I. (1997). Is there a link between engineering and autism? Autism, I(1), 101-109.

Bettelheim B. (1967). The empty fortress: infantile autism and the birth of the self. New York: Free Press.

Bolton, P.F., Macdonald, H., Pickles, A., Rios, P., Goode, S., Crowson, M., et al (1994). A case-control family history study of autism. Journal of Child Psychology and Psychiatry, 35, 877-900.

Bolton, P.F., Murphy, M., Macdonald,H., Whitlock, B., Pickles, A. \& Rutter, M. (1997). Obstetric complications in autism: consequences or causes of the condition? Journal of the American Academy of Child and Adolescent Psychiatry, 36, 272281.

Bolton, P.F., Pickles, A., Murphy, M., \& Rutter, M. (1998). Autistic, affective and other psychiatric disorders: Patterns of familial aggregation. Psychological Medicine, $28,385-395$.

Briskman, J., Happé, F., \& Frith, U. (2001). Exploring the cognitive phenotype of autism: Weak "central coherence" in parents and siblings of children with autism. II. Real-life skills and preferences. Journal of Child Psychology and Psychiatry, $42,309-316$.

Borwnlow, C. \& O'Dell, L. (2002). Ethical issues for qualitative research in on-line communities. Disability \& Society, 17(6), 685-694.

Bureau of Labor Statistics (2000). Most Mothers Work. U.S. Department of Labor. Washington, DC. Retrieved April 12, 2008 from http://www.bls.gov/opub/working/sources.htm . 
Chen, D.T., Miller, F.G. \& Rosenstein, D.L. (2003). Ethical aspects of research into the etiology of autism. Mental Retardation and Developmental Disabilities Research Reviews, 9, 48-53.

Croen, L.A., Grether, J.K. \& Selvin, S. (2002). Descriptive epidemiology of autism in a California population: Who is at risk? Journal of Autism and Developmental Disorders, 32, 217-224.

Cryan, El., Byrne, M., O’Donovan, A. \& O'Callaghan, E. (1996). Brief report: a casecontrol study of obstetric complications and later autistic disorder. Journal of Autism and Developmental Disorders, 26, 453-460.

Dominick, K.C., Davis, N.O. \& Lainhart, J. (2007) Atypical behaviors in children with autism and children with history of language impairment. Research in Developmental Disabilities, 28,145-162.

Duncan, J. (1986). Disorganization of behaviour after frontal lobe damage. Cognitive Neuropsychology, 3, 271-290.

Dyches, T.T., Wilder, L.K. \& Obiakor, F.E. (2001). Autism: multicultural perspectives. In Wahlberg, T., Obiakor, F., Burkhardt.S., et al. (Eds), Educational and Clinical Interventions. (pp 151-177) Oxford, UK: Elsevier Science Ltd.

Eaton, W.W., Mortensen, P.B., Thomsen, P.H. \& Frydenberg, M. (2001). Obstetric complications and risk for severe pathology in childhood. Journal of Autism and Developmental Disorders, 31, 279-285.

Filipek, P., Accardo, P., Baranek, G., Cook, E., Dawson, G., Gordon, B., et al. (1999). The screening and diagnosis of autistic spectrum disorders. Journal of Autism and Developmental Disorders, 29, 349-434. 
Frith, U. (1989). Autism: Explaining the Enigma. Oxford, England: Blackwell.

Fombonne, E. (2005a). The changing epidemiology of autism. Journal of Applied Research in Intellectual Disabilities, 18, 281-294.

Fombonne, E. (2005b). Epidemiological studies of pervasive developmental disorders. In Volkmar, F.R., Paul, R., Klin, A. \& Cohen, D. (Eds.), Handbook of autism and pervasive developmental disorders (pp. 42-69). Hoboken, NJ: John Wiley \& Sons, Inc.

Frith, U. (ed.). (1991). Autism and asperger syndrome. Cambridge, England: Cambridge University Press.

Ghaziuddin, M. (2005). A family history study of asperger syndrome. Journal of autism and developmental disorders, 35, 177-182.

Ghaziuddin, M., Shakal, J. \& Tsai, L. (1995). Obstetric factors in Asperger syndrome: comparison with high-functioning autism. Journal of Intellectual Disability Research, 39, 538-543.

Gillberg, C. \& Cederlund, M. (2005). Asperger Syndrome: Familial and Pre- and Perinatal Factors. Journal of Developmental Disorders, 35, 159-166.

Gillberg, I.C. \& Gillberg, C. (1996). Autism in immigrants: a population-based study from Swedish rural and urban areas. Journal of Intellectual Disability Research, 40, 24-31.

Gillberg, I.C. \& Gillberg, C. (1993). Infantile autism: a total population study of reduced optimality in the pre-, peri- and neonatal period. Journal of Autism and Developmental Disorders, 13, 153-166.

Glasson, E.J., Bower, C., Petterson, B., de Klerk, N, Chaney, G. \& Hallmayer, J.F. 
(2004). Perinatal factors and the development of autism. Arch Gen Psychiatry, 61, $618-627$.

Green, V.A., Pituch, K.A., Itchon, J., Choi, A., O’Reilly, M. \& Sigafoos, J. (2006). Internet survey of treatments used by parents of children with autism. Research in Developmental Disabilities, 27, 70-84.

Goodman, R. \& Richards, H. (1995). Child and adolescent psychiatric presentations of second-generation Afro-Caribbeans in Britain. British Journal of Psychiatry, 167, $362-369$.

Guralnick, M.J. (1998). Effectiveness of early intervention for vulnerable children: A developmental perspective. American Journal of Mental Retardation, 102, 319-45. Happé, F. (2005). The weak central coherence account of autism. In Volkmar, F.R., Paul, R., Klin, A. \& Cohen, D. (Eds.), Handbook of autism and pervasive developmental disorders (pp. 640-649). Hoboken, NJ: John Wiley \& Sons, Inc. Health Canada (2002). Mental illnesses in Canada: An overview. In Stewart, P., Lips, T., Lakaski, C. \& Upshall, P. (Eds), A Report on Mental Illnesses in Canada (pp. 1530). Ottawa, Canada.

Hicks, R., Bowker, A. \& Wells, K. (2006). Treatments for autism: Parental choices and perceptions of change. Unpublished manuscript.

Howlin, P., Goode, S., Hutton, J. \& Rutter, M. (2004). Adult Outcome for children with autism. Journal of Child Psychology and Psychiatry, 45, 212-229.

Human Resources and Social Development (2006). National Occupational Classification. http://www23.hrdc-drhc.gc.ca/2001/e/groups/index.shtml

Jones, M.B. \& Szatmari, P. (1988). Stoppage rules and genetic studies of autism. Journal 
of Autism and Developmental Disorders, 18, 31-40.

Joseph, K.S., Kramer, M.S., Marcoux, S., Ohlsson, A., Wen, S.W., Allen, A. et al. (1998). Determinants of preterm birth rates in Canada from 1981 through 1983 and from 1992 through 1994. New England Journal of Medicine, 229, 1434-1439.

Kanner, L. (1943). Autistic disturbances of affective contact. Nervous child, 2, 217-250.

Kessler RC, Chiu WT, Demler O, Walters EE. (2005). Prevalence, severity, and comorbidity of twelve-month DSM-IV disorders in the National Comorbidity Survey Replication (NCS-R). Archives of General Psychiatry, 62(6), 617-627.

Klin, A., Mcpartland, J. \& Volkmar, F.R. (2005). Asperger Syndrome. In Volkmar, F.R., Paul, R., Klin, A. \& Cohen, D. (Eds.), Handbook of autism and pervasive developmental disorders (pp. 88-125). Hoboken, NJ: John Wiley \& Sons, Inc

Klug, M.G., Burd, L., Kerbeshian, J., Benz, B. \& Martsolf, J.T. (2003). A comparison of the effects of parental risk markers on pre- and perinatal variables in multiple patient cohorts with fetal alcohol syndrome, autism, Tourette syndrome, and sudden infant death syndrome: an enviromic analysis. Neurotoxicology and Teratology, 25, 707-717.

Landa, R. \& Garrett-Mayer, (2006). Development in infants with autism spectrum disorders: a prospective study. Journal of Child Psychology and Psychiatry, 47, 629-638.

Lauritsen, M.B., Pedersen, C.B. \& Martensen, P.B. (2005). Effects of familial risk factors and place of birth on the risk of autism: a nationwide register-based study. Journal of Child Psychology and Psychiatry, 46, 963-971.

Limperopoulos, C., Basson, H., Sullivan, N.R., Soul, J.S., Robertson, Jr., Moore, M., 
Ringer, S., Vope, J.J. \& Du Plessis, A.J. (2008). Positive screening for autism in ex-preterm infants: Prevalence and Risk Factors. Pediatrics, 121(4), 758-765.

Li, S., Li, X., Zhang, X., Luo, X., Su, L., Che, J. \& Yan, J. (2002). Risk factors of childhood autism and manifestation in early stage. Chinese Mental Health Journal, 16, 552-554.

Liu, X. et al. (2005). Brief Report: An epidemiologic survey of the prevalence of sleep disorders among children 2 to 12 years old in Beijing, China. Pediatrics, 115, 26608151.

Lovaas, O.I. (1987). Behavioral treatment and normal educational and intellectual functioning in young autistic children. Journal of Consulting and Clinical Psychology, 55, 3-9.

Maimburg, R.D. \& Vaeth, M. (2006). Perinatal risk factors and infantile autism. Acta Psychiatrica Scandinavica, 114, 257-264.

Matson, J.L. \& LoVullo (2008). A review of behavioral treatments for self-injurious behaviors of persons with autism spectrum disorders. Behavior Modification, $32(1), 61-76$.

Matsuishi, T., Yamashita, Y., Ohtani, Y., Ornitz, Ed., Kuriya, N. Yoshihiko, M. et al. (1999). Brief report: incidence of and risk factors for autistic disorder in neonatal intensive care unit survivors. Journal of Autism and Developmental Disorders, $29,161-166$.

Matthews, M.S. \& Hamilton, B.E. (2002). Mean age of mother. National Vital Statistics Reports, 51(1). Retrieved April 17, 2008 from http://www.ncbi.nlm.nih.gov/pubmed/12564162. 
McClintock, K., Hall, S. \& Oliver, C. (2003). Risk markers associated with challenging behaviours in people with intellectual disabilities: a meta-analytic study. Journal of Intellectual Disability Research, 47, 405-416.

Mercer, L., Creighton, S., Holden, J.J.A. \& Lewis, M.E.S. (2006). Parental perspectives on the causes of an autism spectrum disorder in their children. Journal of Genetic Counseling, 15, 41-50.

Micali, N., Chakrabarti, S. \& Fombonne, E. (2004). The broad autism phenotype: Findings from an epidemiological survey. Autism, 8, 21-37.

Minde, K, Popiel K, Leos N, Falkner S, Parker K, Handley-Derry M. (1993). The evaluation and treatment of sleep disturbance in young children. Journal of Child Psychology and Psychiatry, 4, 521-533.

Mindel, J.A., Owens, J.A., Carskadon, M.A. (1999). Developmental features of sleep. Child and Adolescent Psychiatric Clinics of North America, 8, 695-725.

Mouridsen, S.E., Rich, B., Isager, T. \& Nedergaard, N.J. (2007). Psychiatric disorders in the parents of individuals with infantile autism: a case-control study. Psychopathology, 40, 166-171.

Mundy, P. \& Burnette, C. (2005) Joint attention and neurodevelopmental models of autism. In Volkmar, F.R., Paul, R., Klin, A. \& Cohen, E. (Eds) Handbook of autism and pervasive developmental disorders (pp. 650-681). New Jersey, John Wiley \& Sons, Inc.

Murphy, M., Bolton, P., Pickles, A., Fombonne, El, Piven, J., \& Rutter, M. (2000). Personality traits in the relatives of autistic probands. Psychological Medicine, 30, 1411-1424. 
National Center for Health Statistics. (2007). Births: Final Data for 2005. National Vital Statisitics Reports, 56(6). U.S. Dept. of Health and Human Services.

New York State Department of Health Early Intervention Program (1999). Clinical practice guidelines: Report of the recommendations on autism and pervasive developmental disorders. New York: State Department of Health. Retrieved March 23, 2005 from www.health.state.ny.us/nysdoh/eip/menu.htm .

O'Hare, A., Whelan, C.T., \& Commins, P. (1991). The development of an Irish censusbased social class scale. Economic and Social Review, 22, 135-156.

Osterling, J., Dawson, G., \& Munson, J. (2002). Early recognition of 1 -year-old infants with autism spectrum disorder versus mental retardation. Developmental and Psychopathology, 14, 239-251.

Ozonoff, S., South, M. \& Provencal, S. (2005) Executive Functions. In Volkmar, F.R., Paul, R., Klin, A. \& Cohen, E. (Eds) Handbook of autism and pervasive developmental disorders (pp. 606-627). New Jersey, John Wiley \& Sons, Inc.

Reichenberg, A., Gross,R., Weiser, M., Bresnaham, M., Silverman, J., Harlap, S. et al. (2006). Advancing paternal age and autism. Arch Gen Psychiatry, 63, 1026-1032.

Rodier, P.M. \& Hyman, S.L. (1998). Early environmental factors in autism. Mental Retardation and Developmental Disabilities, 4, 121-128.

Rogers, S.J. (1998). Empirically supported comprehensive treatments for young children with autism. Journal of Clinical Child Psychology, 27, 168-179.

Rutter, M. (2005a). Aetiology of autism: findings and questions. Journal of Intellectual Disability Research, 49, 231-238. 
Rutter, M. (2005b). Genetic influences and autism. In Volkmar, F.R., Paul, R., Klin, A. \& Cohen, E. (Eds) Handbook of autism and pervasive developmental disorders (pp. 425-452). New Jersey, John Wiley \& Sons, Inc.

Schroeder, S.R., Schroeder, C.S., Smith, B. \& Dalldord, J. (1978). Prevalence of selfinjurious behaviors in a large state facility for the retarded: a three-year follow-up study. Journal of Autism and Childhood Schizophrenia, 8, 261-269.

Smith, T. (1999). Outcome of early intervention for children with autism. Clinical Psychology: Science and Practice, 6, 33-49.

Statistics Canada (2006). Canadian labour market at a glance: Employment rates of mothers, by age of youngest child. Labour Force Survey. Retrieved April 12, 2008 from www.statcan.ca/english/freepub/71-222-XIE/2006001/chartb10.htm.

Szatmari, P., MacLean, J.E., Jones, M.B., Bryson, S.E., Zwaigenbaum, L., Bartolucci, G., et al. (2000). The familial aggregation of the lesser variant in biological and nonbiological relatives of PDD probands: A family history study. Journal of Child Psychology and Psychiatry, 41, 579-586.

Tharp, B. (2003). Contributions of neurology. In Ozonoff, S., Rogers, S. \& Hendren, R. (Eds) Autism spectrum disorders: A research review for practitioners (pp. 11129). VA: American Psychiatric Publishing, Inc.

Tidmarsh, L. \& Volkmar, F.R. (2003). Diagnosis and epidemiology of autism spectrum disorders. Canadian Journal of Psychiatry, 48, 517-525.

Tsai, L. \& Steward, M.A. (1983). Etiological implication of maternal age and birth order in infantile autism. Journal of autism and developmental disorders, 13, 57-65. 
Volkmar, F.R. \& Pauls, D. (2003). Autism. Lancet, 362, 1133-41.

Volkmar, F.R. \& Rutter, M. (1995). Childhood disintegrative disorder: Results of the DSM-IV Autism Field Trial. Journal of the American Academy of Child and Adolescent Psychiatry. 34(8), 1092-1095.

Wiggs, L. \& Stores, G. (2004). Sleep patterns and sleep disorders in children with autistic spectrum disorders: insights using parent report and actigraphy. Developmental Medicine \& Child Neurology, 46, 372-380.

Williams, G., Oliver, J.M., Allard, A. \& Sears, L. (2003). Autism and associated medical and familial factors: A case control study. Journal of Developmental and Physical Disabilities, $15,335-349$.

Wing, L. (1980). Childhood autism and social class: a question of selection? British Journal of Psychiatry, 137, 410-417.

Wing, L. \& Potter, D. (2002). The epidemiology of autistic spectrum disorders: is the prevalence rising? Mental Retardation and Developmental Disabilities Research Review, 8, 151-161.

Wodrich, D.L. (1997). Children's Psychological Testing: A Guide for Nonpsychologists ( $3^{\text {rd }}$ Ed.). Baltimore: Paul H. Brookes Publishing Co.

World Health Organization (1993). The International Statistical Classification of Diseases and Related Health Problems, $10^{\text {th }}$ Revision (ICD-10). Geneva: Switzerland.

Zwaigenbaum, et al. (2002). Pregnancy and birth complications in autism and liability to the broader autism phenotype. Journal of the American Academy of Child $\&$ Adolescent Psychiatry, 41, 572-579. 
Appendix A

Internet Survey

By completing this survey it is assumed that you have given your consent for the researchers to use the information as required. The survey is completely anonymous and under no circumstances will you be identified by name or have your personal information shared. The information collected will only be used for research purposes. Please omit any questions which make you feel uncomfortable and /or you would rather not answer.

When this survey refers to "your child" or "your son/daughter" we are referring to the child diagnosed with an autism spectrum disorder (ASD), unless otherwise specified. Should you have two or more children with an ASD it would be greatly appreciated if you could fill out one survey for each child. Thank you very much for your participation. For each question please circle the response(s) that best apply.

\section{General Information}

1. What is the sex of your child? Male Female

2. What is his/her birth date (year/month/day)?

3. How many children do you have (including the child(ren) with ASD)?

$$
\begin{array}{llllll}
1 & 2 & 3 & 4 & 5 & +5
\end{array}
$$

4. If you have more than one child, please answer the following:

\begin{tabular}{|c|c|c|c|c|}
\hline & \multicolumn{3}{|c|}{ SEX } & Date of Birth \\
\hline Sibling \#1 & & or & $\mathrm{F}$ & \\
\hline Sibling \#2 & $\overline{\mathrm{M}}$ & or & $F$ & \\
\hline Sibling \#3 & $\bar{M}$ & or & $\bar{F}$ & \\
\hline Sibling \#4 & $\overline{\mathrm{M}}$ & or & $\bar{F}$ & \\
\hline
\end{tabular}

5. What is the father's birth date (year/month/day)?

6. What is the mother's birth date (year/month/day)? 
7. Where do you currently reside? (please indicate City and Province/State only)

8. What services are available for you to access?
a) community services
b) financial assistance
c) hospital-based services
d) occupational therapy
e) speech therapy
f) physical therapy
g) special education
h) other (please specify)

\section{Diagnosis}

9. How old was your child when he/she was diagnosed?

10. What diagnosis did your child receive?
a) PDD-NOS
b) Autistic Disorder
c) Asperger's Disorder
d) Other (please specify)

11. What instruments were used to diagnose your son/daughter? 
12. If the diagnosis was based (at least in part) on clinical observation, how long was your child observed for (round to the closest full hour)?
a) 5 or less hours
b) 6-10 hours
c) $11-15$ hours
d) 16-20 hours
e) 21 or more hours

13. Has your child's diagnosis changed since the original diagnosis?

Yes no (if no, skip to question 15)

14. If yes, what is their current diagnosis?
a) PDD-NOS
b) Autistic Disorder
c) Asperger's Disorder
d) other (please specify)

\section{Family History}

15. Are there any other cases of ASDs in your family?

Yes no (if no, skip to question 17)

16. If yes, what is the relation of that person to your son/daughter?
a) mother
b) father
c) sibling
d) grandmother
e) grandfather 
f) first cousin

g) second cousin

h) aunt

i) uncle

j) other (please specify)

17. Are there any cases of other mental disorders in your family (either immediate or extended)?

Yes No (if no, skip to question 19)

18. If yes, please indicate the disorder, along with the affected individual's relationship to your child (including which side of the family he/she is on) in the following chart:

\begin{tabular}{|l|c|c|}
\hline Relationship to Child & $\begin{array}{l}\text { Mother's (Mo) or Father's (Fa) side } \\
\text { of the family (circle Mo or Fa) }\end{array}$ & Disorder(s) \\
\hline & Mo OR Fa & \\
\hline Mo OR Fa & \\
\hline Mo OR Fa & \\
\hline Mo OR Fa & \\
\hline
\end{tabular}

19. What is the mother's occupation?

20. What is the father's occupation?

21. What is the household's average annual income?

a) less than $\$ 20,000$

b) 20,000 to 40,000 

c) $40,000-60,000$
d) $60,000-80,000$
e) above 80,000

22. What cultural background does your family come from? (e.g. Italian, Asian, Irish, etc.)

\section{Medical History}

23. Were there any complications during the pregnancy or delivery of your child? (e.g breech birth, excessive bleeding) yes no (if no, ski; to question 25)

24. If yes, what were the complications?

25. At what week of gestation was your child born?

26. Does your child display any self-injurious behaviours?

Yes No

27. If yes, what are they?

28. When do they occur? (please select as many that apply)
a) when frustrated
b) unprovoked
c) when excited
d) when under stimulated
e) randomly
f) other (please specify) 
29. How many times per day do the behaviour(s) occur?
a) less than 1
b) $1-5$
c) 6-10
d) $11-15$
e) $16-20$
f) over 20

30. How severe are these behaviours (state, for example, if they leave bruises, marks, is hospitalization required, have they cause permanent physical damage, etc.)?

31. Does your child engage in any self-stimulatory behaviour?

Yes No

32. If yes, what are the behaviours that your child engages in? For each behaviour please indicate which sensory mechanism is being stimulated. In other words, is it a verbal, visual, oral, tactile (feel), gross motor, movement/motion, or olfactory (smell) stimulatory behaviour?

33. When do the behaviour(s) occur? (please select as many that apply)
a) when frustrated
b) unprovoked
c) when excited
d) when under stimulated 

e) randomly
f) other (please specify)

34. How many times per day do the behaviour(s) occur?
a) less than 1
b) $1-5$
c) 6-10
d) $11-15$
e) $16-20$
f) over 20

35. What form of communication does your child use?
a) verbal
b) sign
c) picture exchange
d) other (please specify)

36. Does your daughter/son have any allergies or sensitivities?

Yes no (if no, skip to question 38)

37. If so, what are they?

38. Does your daughter/son have any other medical conditions or problems?

Yes No (if no, skip to question 40)

39. If yes, what are they?

40. Does your child display any sleep disorders?

Yes No (if no, skip to question 42) 
41. If yes, what kinds of sleep problems does your child display (please circle all that apply)?
a) apnea
b) awakenings
c) night terrors
d) irregular sleeping times
e) other (please specify)

42. Has your daughter/son ever stayed overnight at a hospital?

Yes no (if no, skip to question 45)

$\begin{array}{llllllll}\text { 43. If yes, how many times? } & 1 & 2 & 3 & 4 & 5 & 6 & +7\end{array}$

44. For what reason(s) was the hospital stay necessary?

45. Has your child ever been taken to an emergency room?

Yes no (if no, skip to question 48)

$\begin{array}{llllllll}\text { 46. If yes, how many times? } & 1 & 2 & 3 & 4 & 5 & 6 & +7\end{array}$

47. For what reason(s) did your child go to the emergency room?

48. Has your child ever undergone a neurological exam? (for example, MRI, CAT, etc.)

Yes No (if no, skip to question 50)

49. If yes, at what age was the test taken, and what were the results?

50. Has your child ever undergone an IQ assessment/intelligence test?

Yes No (if no skip to question 50) 
51. If so, at what age and what were the results?

52. In your opinion, what is the most salient feature of the disorder in your son/daughter? In other words what is the single most ASD characteristic that your child typifies (circle the one that best applies)?
a) self-stimulatory behaviours
b) self-injurious behaviours
c) echololia
d) fixations
e) rigidity/inflexibility
f) lack of social connectedness
g) other (please specify)

\section{(Treatment questions \#53-72 omitted)}

Thank you for your participation in this study. It is hoped that this research will add to the body of autism literature a complete and accurate representation of the autistic population. In doing so we hope to stimulate further research in the area and at the same time gain a better idea of the disorder itself. As soon as the results have been collected, we will release them to your organization, or if you would like a copy please contact us. If you have any other comments, questions, or concerns please feel free to contact:

Kerry Wells at: 613-520-2600 or rlw27@hotmail.com Thank you again for your time and participation. 


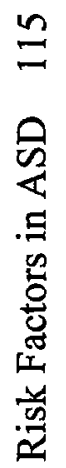

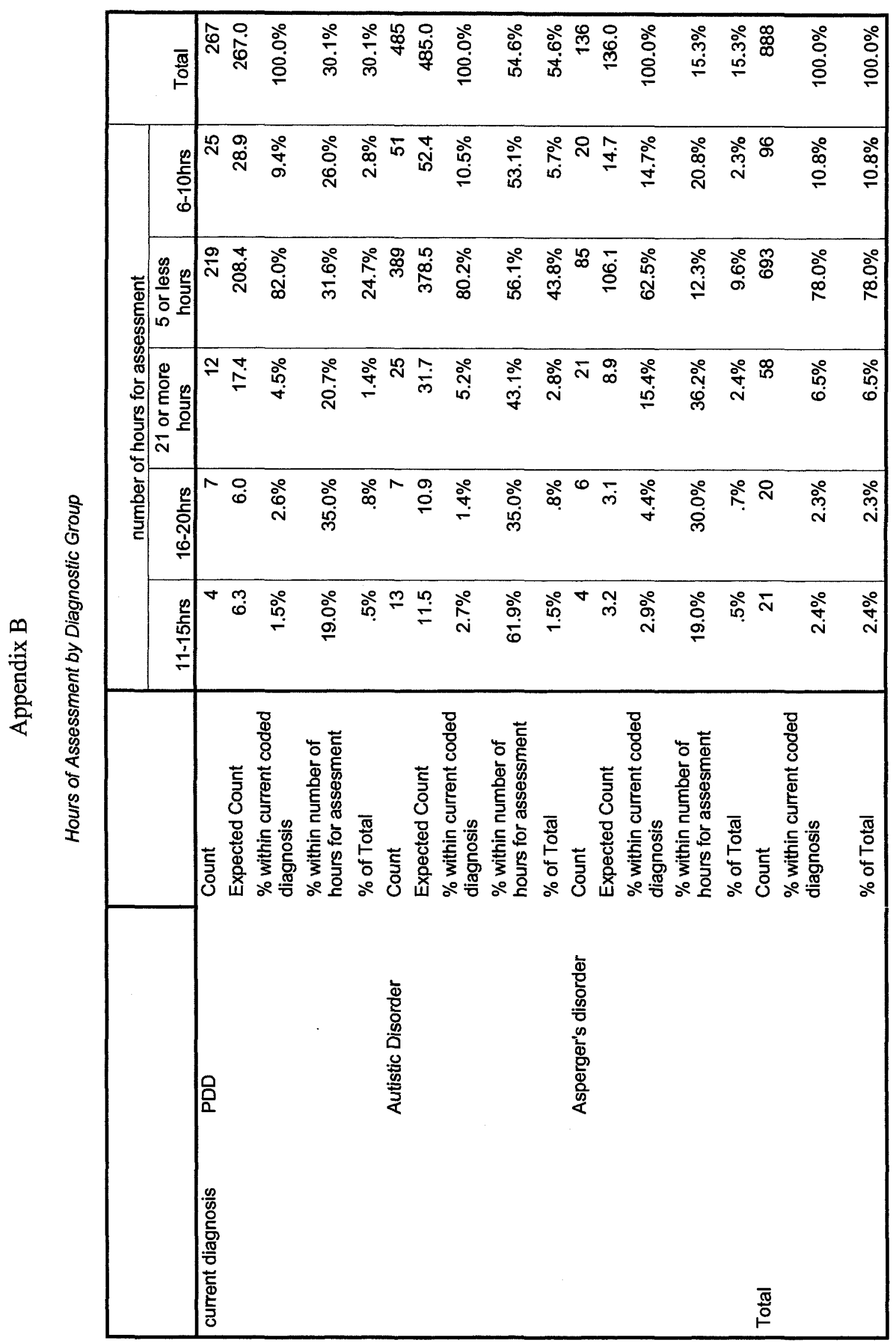


0
0
0
0
$\vdots$
0
0
0
0
0
0
0
0
0

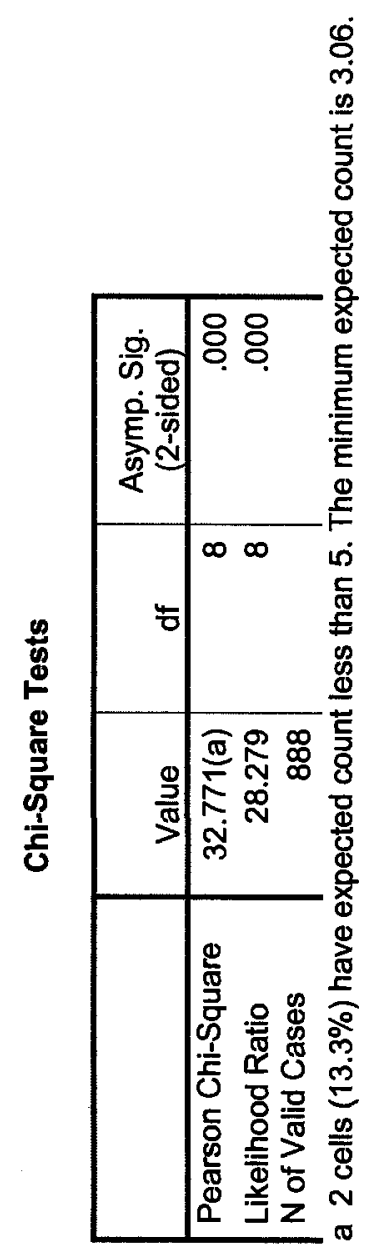




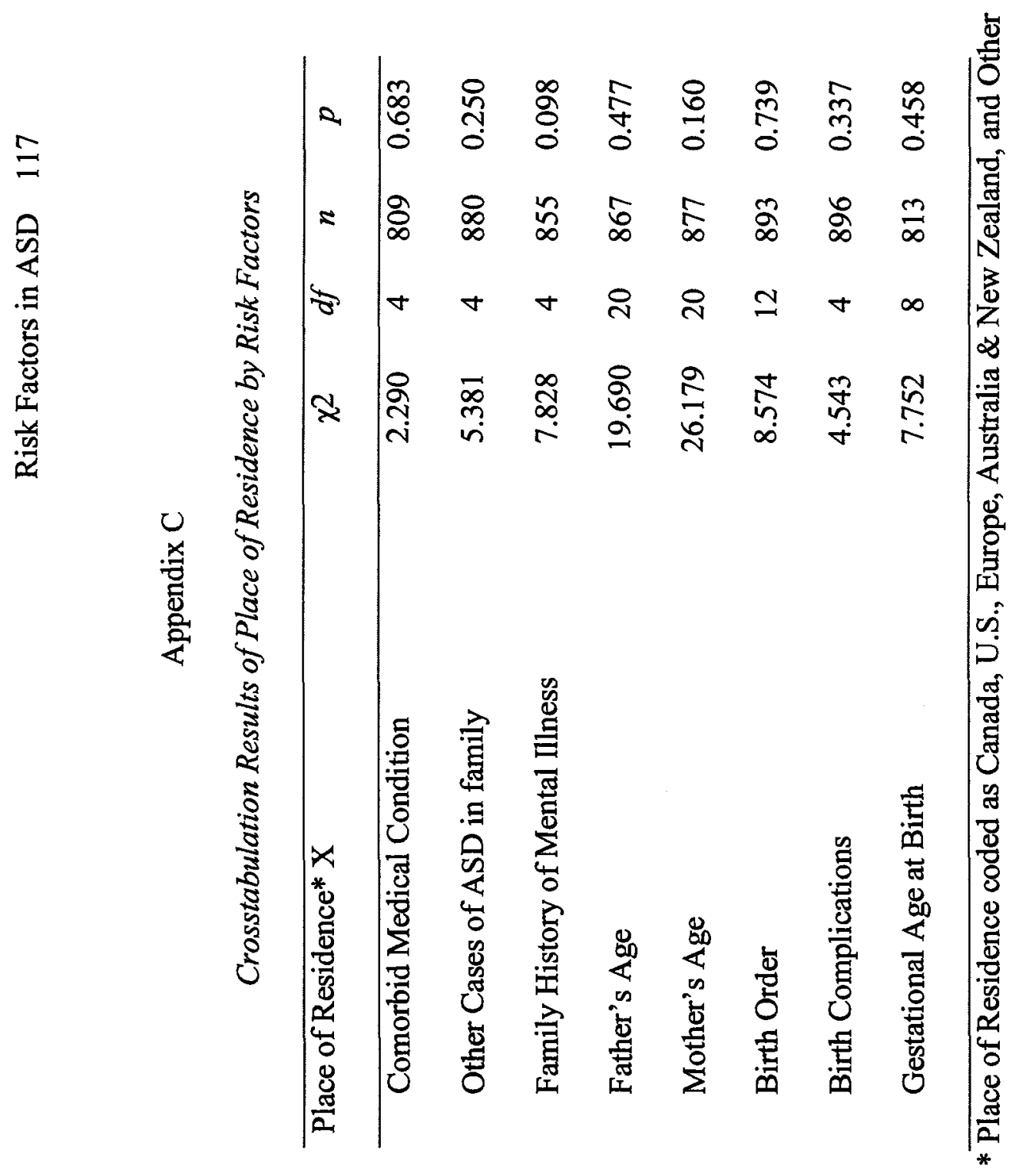




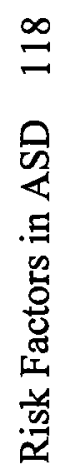

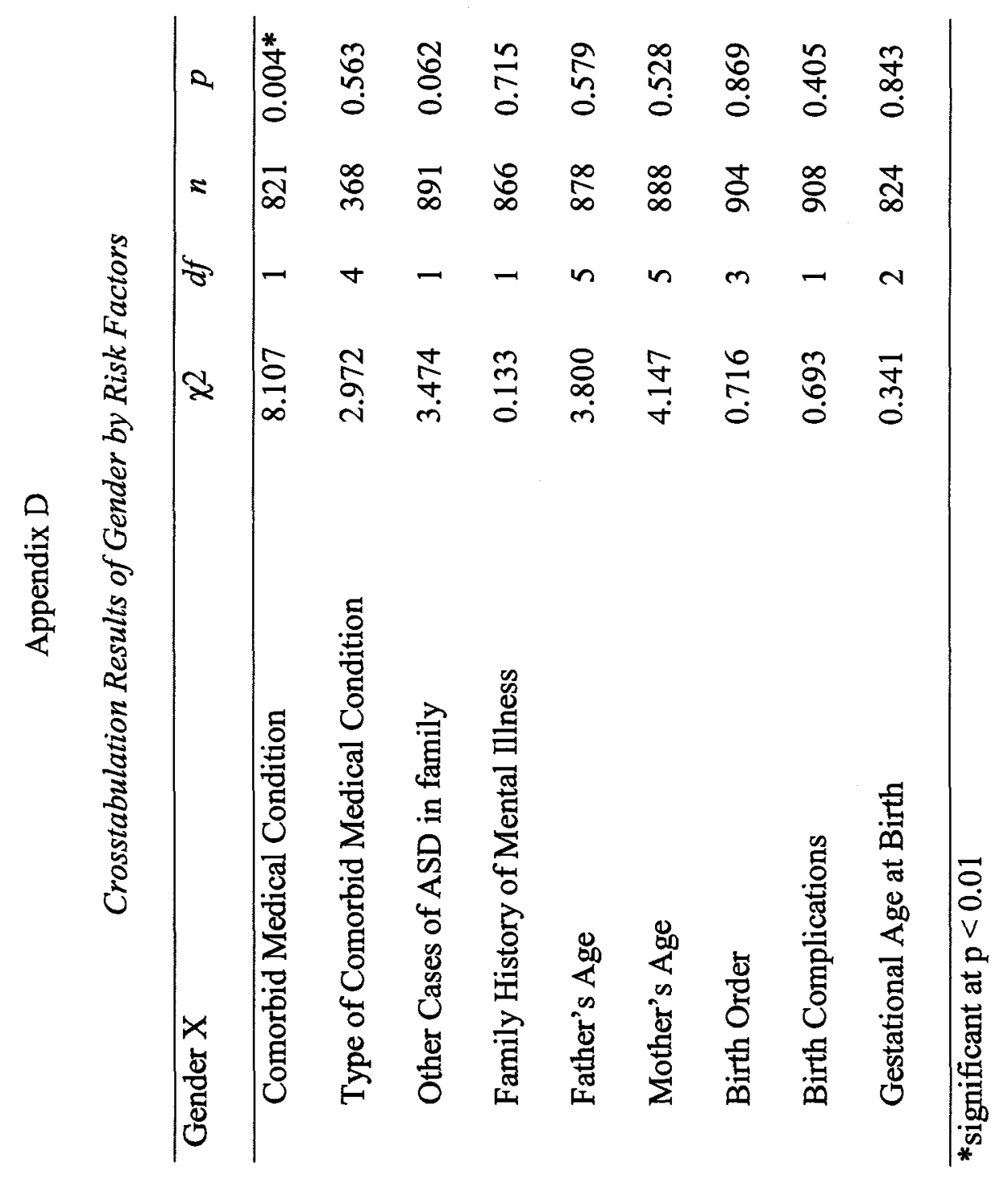




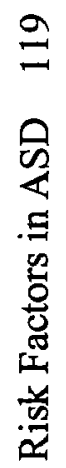

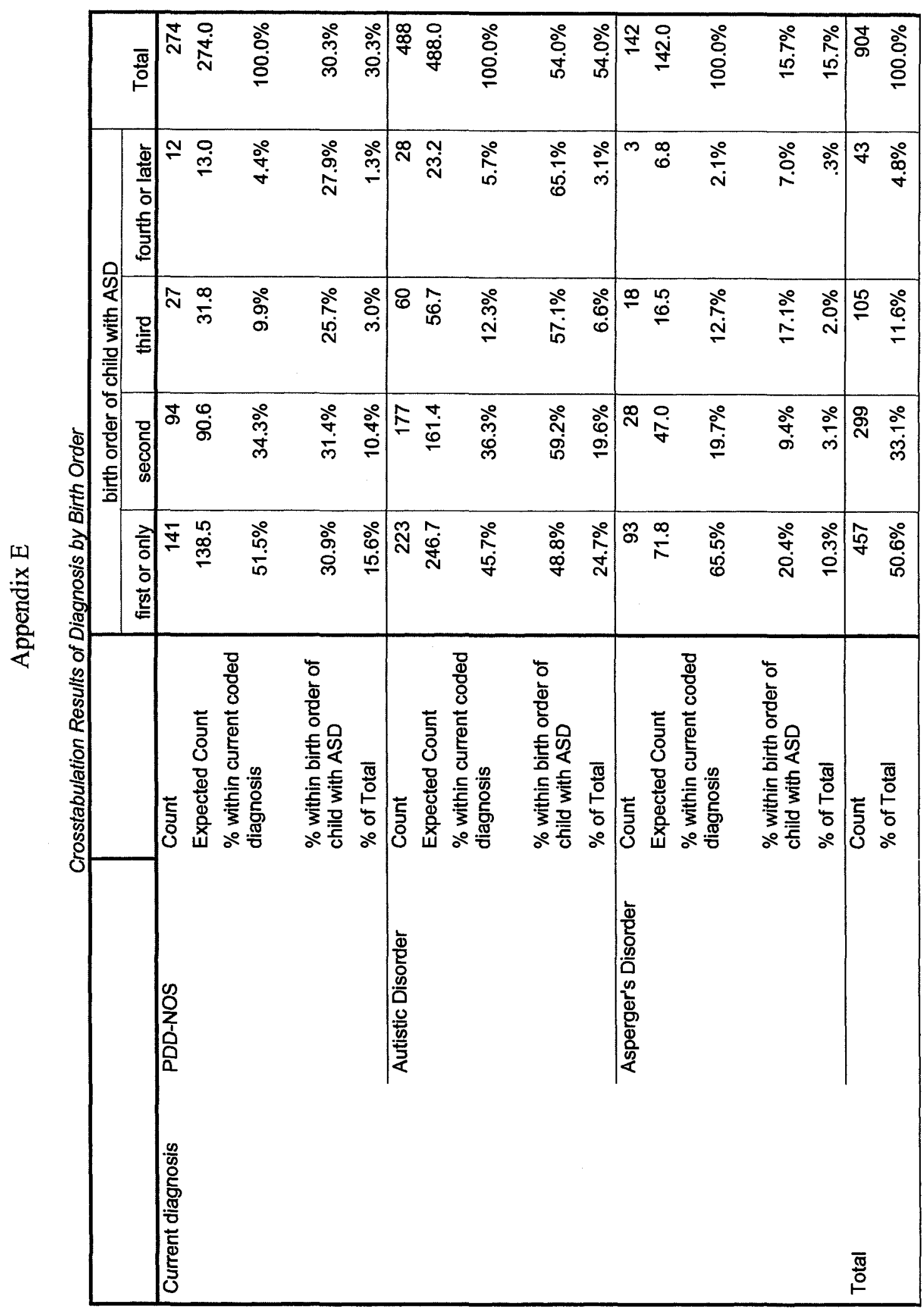




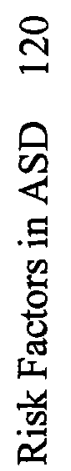

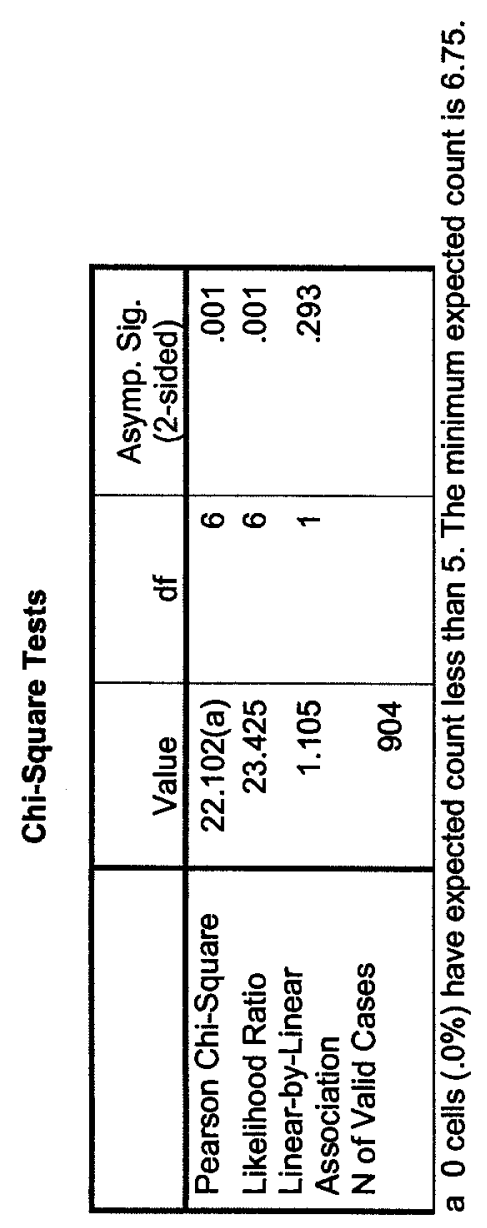

